MATHEMATICS OF COMPUTATION

Volume 68, Number 228, Pages 1465-1496

S 0025-5718(99)01108-4

Article electronically published on May 21, 1999

\title{
ALMOST OPTIMAL CONVERGENCE OF THE POINT VORTEX METHOD FOR VORTEX SHEETS USING NUMERICAL FILTERING
}

\author{
RUSSEL E. CAFLISCH, THOMAS Y. HOU, AND JOHN LOWENGRUB
}

\begin{abstract}
Standard numerical methods for the Birkhoff-Rott equation for a vortex sheet are unstable due to the amplification of roundoff error by the Kelvin-Helmholtz instability. A nonlinear filtering method was used by Krasny to eliminate this spurious growth of round-off error and accurately compute the Birkhoff-Rott solution essentially up to the time it becomes singular. In this paper convergence is proved for the discretized Birkhoff-Rott equation with Krasny filtering and simulated roundoff error. The convergence is proved for a time almost up to the singularity time of the continuous solution. The proof is in an analytic function class and uses a discrete form of the abstract Cauchy-Kowalewski theorem. In order for the proof to work almost up to the singularity time, the linear and nonlinear parts of the equation, as well as the effects of Krasny filtering, are precisely estimated. The technique of proof applies directly to other ill-posed problems such as Rayleigh-Taylor unstable interfaces in incompressible, inviscid, and irrotational fluids, as well as to Saffman-Taylor unstable interfaces in Hele-Shaw cells.
\end{abstract}

\section{INTRODUCTION}

Standard numerical methods are generally not convergent for ill-posed problems. Typically, in an ill-posed problem, the linear growth rates increase unboundedly with increasing wavenumber. Such problems may have short time smooth solutions if the Fourier coefficients of the initial data have rapid enough decay (i.e., existence in analytic function spaces $[5,12,23])$. However, when standard numerical methods are used to compute them, the methods prove to be highly unstable. This is because, on the numerical level, the decay of the Fourier coefficients is limited by the numerical precision. For example, the Fourier coefficients of the initial data decay only until the roundoff level is reached. Roughly speaking, all subsequent modes are dominated by roundoff error and do not decay. Since these highest modes are amplified the fastest in time, the numerical solution becomes dominated

Received by the editor December 16, 1997.

1991 Mathematics Subject Classification. Primary 65M25; Secondary 76C05.

Key words and phrases. Vortex sheets, point vortices, numerical filtering, discrete CauchyKowalewski theorem.

The first author's research was supported in part by the Army Research Office under grants \#DAAL03-91-G-0162 and \#DAAH04-95-1-0155, the second author's by ONR Grant N00014-961-0438 and NSF Grant DMS-9704976, and the third author's by the McKnight Foundation, the National Science Foundation, the Sloan Foundation, the Department of Energy, and the University of Minnesota Supercomputer Institute.

(C)1999 American Mathematical Society 
by spurious error and the computation breaks down, even though the true solution may still be very smooth.

A prototypical ill-posed problem, and the one we will consider in this paper, is the evolution of a vortex sheet in an incompressible, inviscid, and otherwise irrotational fluid. This is a classical problem in fluid dynamics, and the sheet undergoes the Kelvin-Helmholtz instability. In this problem, the linear growth rate is proportional to the wavenumber of the initial perturbation. Moreover, singularity formation appears to be generic, even for vortex sheets initially near equilibrium $[17,15,6,22,9]$. One motivation for performing numerical simulations of the vortex sheet problem is to characterize the types of singularities that can form and to determine whether there is in fact a "generic" type. See [9] for a very recent and thorough study of singularity formation and evolution for the vortex sheet problem.

To accurately compute the numerical evolution of a vortex sheet, one must overcome the spurious growth of roundoff error. This can be done using a numerical filter. However, standard linear filters, such as removing, or damping, a fixed band of modes, often "over-smooth" the details of the solution, making singularity characterization difficult. Moreover, through nonlinearity, the physically relevant spectrum typically expands in time into the region of artificially removed wavenumbers. If this region is fixed independently of the discretization parameters and of time, then this type of filtering scheme will no longer converge at such times. On the other hand, a nonlinear filtering, introduced to this problem by Krasny [15], has proven very successful. The Krasny filter sets equal to zero all Fourier modes lying below a certain error tolerance and leaves those lying above the tolerance unchanged. The filter is nonlinear, because the modes it removes depend on the function to which the filter is applied. Important consequences of this filter are that it allows nonlinearity to produce non-zero modes anywhere in the spectrum, and that the linear growth rate is determined by the discretization and not the filter. Using this nonlinear filter, Krasny [15] and subsequently Shelley [22] were able to accurately compute numerical solutions essentially up to the time they become singular.

In this paper we prove that in the presence of simulated roundoff error and Krasny filtering, the point vortex method (PVM) and the spectrally accurate modified point vortex method (MPVM [22]) both converge to the solution of the BirkhoffRott equation. The proof is in an analytic function class and uses a discrete form of the Cauchy-Kowalewski theorem [7, 18, 19, 21]. The proof is presented for the case in which the sheet is initially near equilibrium and convergence is obtained nearly up to the singularity time. This result is nearly optimal, and is referred to as a "long time" convergence theorem. This is a significant improvement over previous convergence theorems for this problem, where the time of convergence was restricted to be much less than the singularity time $[8,14]$. The near equilibrium case was studied on the continuous level in $[5,23]$. If the near equilibrium condition is violated, convergence is obtained for a short time provided the true solution remains smooth.

The improved result rests on two observations. First, the nonlinear filter must be included in the analysis to control the growth of the round-off error in time. We note that the previous convergence results did not include the nonlinear filter, as the analysis of it was incomplete at that time. Still, this is not enough to obtain a "long time" convergence theorem. Second, it also is necessary to separate the linear and nonlinear parts of the equation. Both parts of the equation must then 
be precisely estimated. This is analogous, in spirit, to the continuous analysis of [5], where the linear part of the equation is solved exactly (by integration along complex characteristics) and precise bounds were obtained for the nonlinear operator. The analysis in this paper applies directly to other numerical methods, such as the modified point vortex method [22], as well as to other ill-posed problems, such as Rayleigh-Taylor unstable interfaces in incompressible, inviscid, and irrotational fluids, as well as to Saffman-Taylor unstable interfaces in Hele-Shaw cells. Further, if surface tension is included so that the problems are well-posed $[2,3]$, then the analysis of Krasny filtering presented here, combined with the analysis presented in [4], can be used to prove convergence in that case also.

The outline of the paper is as follows. In Section 2, the nonlinear filtering is introduced and a sequence of model equations is analyzed, providing an overall framework for our analysis. In Section 3, the vortex sheet problem, the PVM and the MPVM are introduced, and the convergence theorem is given. In Section 4, the discrete Cauchy-Kowalewski theorem is presented. In Section 5, the main convergence theorem is proved. In Section 6, the discrete Cauchy-Kowalewski theorem with filtering and roundoff error is proved. A version of the abstract Cauchy-Kowalewski

theorem for continuous time is presented in Appendix 1. Several technical results are deferred to the other two appendices.

\section{NONLINEAR FILTERING AND MODEL PROBLEMS}

The nonlinear filter introduced by Krasny [15] can be considered as a projection operator in Fourier space. It is described as follows. Given an error tolerance $\tau$, the projection operator $P$ is given by

$$
(\widehat{P f})_{k}= \begin{cases}\hat{f}_{k}, & \text { if }\left|\hat{f}_{k}\right| \geq \tau \\ 0, & \text { if }\left|\hat{f}_{k}\right|<\tau\end{cases}
$$

If $f$ is a discrete function, then $\hat{f}_{k}$ is the $k$-th mode of the discrete Fourier transform of $f$. If $f$ is a continuous function, then the continuous Fourier transform is used. The continuous version of Krasny filtering is used in this section for illustration only. In all subsequent sections, $P$ is defined through the discrete Fourier transform.

The filter $P$ is nonlinear because the wavenumbers at which it is applied depend on $f$ itself. This filtering method is most effective when $\hat{f}_{k}$ is rapidly decaying, i.e., the function $f$ is periodic and analytic. It also requires high precision computations, since the filter level $\tau$ must be much larger than the round-off error size $\varepsilon_{r}$. Typical sizes for a double precision computation are $\varepsilon_{r}=10^{-15}, \tau=10^{-12}$.

The usefulness of this nonlinear filtering is that while it prevents the spurious growth of round-off error, it allows the linear growth rate to be determined by the numerical discretization rather than the filtering scheme, since the filter leaves those modes that lie above the tolerance level unchanged. This is most effective for nonlinear problems, because wavenumbers grow due to both linear and nonlinear interactions.

In this section, we present a sequence of examples that show the essential effects of filtering, the necessity of using the abstract Cauchy-Kowalewski theorem and the overall strategy of our convergence proof. We begin with a linear example. 
Consider the simple model equation

$$
\left\{\begin{array}{l}
u_{t}=\mathcal{H}\left(u_{\alpha}\right), \\
u(\alpha, 0)=u_{0}(\alpha)
\end{array}\right.
$$

in which $\mathcal{H}$ is the Hilbert transform; i.e., $(\widehat{\mathcal{H} u})_{k}=-i \operatorname{sgn}(k) \hat{u}_{k}$. Actually, $(2.2)$ arises from the linearization of the equations of motion of an interface in an unstably stratified Hele-Shaw flow in the absence of surface tension [20]. Take the initial data to be $\hat{u}_{0}(k)=e^{-|k| \rho_{0}}$, so that the solution is

$$
\hat{u}_{k}(t)=e^{-|k|\left(\rho_{0}-t\right)} .
$$

This solution develops a singularity at time $T_{0}=\rho_{0}$, when the exponential decay of the Fourier components is lost. Of course, this singularity was "built into" the initial condition.

Now suppose that the initial data is perturbed by simulated roundoff error, and solve equation (2.2) both with and without filtering. For simplicity, we will suppose there is no roundoff error in the equation. This will make the effect of filtering clearer. Moreover, because the equation is linear, the analysis of roundoff error in the equation essentially reduces to that given below for the case when initial data is perturbed by roundoff error. This is because, in the periodic case where $k$ is an integer, multiplication by $|k|$ ensures that if the initial data at mode $k$ lies above the roundoff, then mode $k$ lies above the roundoff at all subsequent times.

The roundoff error is simulated by a perturbation $e_{r}$ with $\hat{e}_{r}(k)=\varepsilon_{r}$ in each Fourier mode (with $\varepsilon_{r} \approx 10^{-15}$ ). The perturbed problem without filtering is

$$
\left\{\begin{array}{l}
\partial_{t} \hat{v}_{k}=|k| \hat{v}_{k}, \\
\hat{v}_{k}(0)=e^{-|k| \rho_{0}}+\varepsilon_{r},
\end{array}\right.
$$

which has solution

$$
\hat{v}_{k}(t)=e^{-|k|\left(\rho_{0}-t\right)}+\varepsilon_{r} e^{|k| t} .
$$

Notice that the initial roundoff error is amplified exponentially in time with a rate proportional to $|k|$.

Now consider the perturbed problem with filtering. It is given by

$$
\begin{aligned}
\partial_{t} \hat{w}_{k} & =P\left\{\widehat{\mathcal{H}\left(w_{\alpha}\right)}\right\}_{k}, \\
\hat{w}_{k}(0) & =\left(P\left\{\widehat{u_{0}+e_{r}}\right\}\right)_{k} \\
& = \begin{cases}e^{-|k| \rho_{0}}+\varepsilon_{r} & \text { if } e^{-|k| \rho_{0}}+\varepsilon_{r} \geq \tau, \\
0 & \text { if } e^{-|k| \rho_{0}}+\varepsilon_{r}<\tau,\end{cases}
\end{aligned}
$$

where, for simplicity, we assume that $\varepsilon_{r}>0$. The solution $\hat{w}_{k}(t)$ to (2.6) is given by

$$
\hat{w}_{k}(t)= \begin{cases}e^{-|k|\left(\rho_{0}-t\right)}+\varepsilon_{r} e^{|k| t} & \text { if } e^{-|k| \rho_{0}}+\varepsilon_{r} \geq \tau, \\ 0 & \text { if } e^{-|k| \rho_{0}}+\varepsilon_{r}<\tau .\end{cases}
$$

Moreover, if $\varepsilon_{r}<\tau$, then $e^{-|k| \rho_{0}}+\varepsilon_{r} \geq \tau$ only for $|k| \leq k_{f}$, where

$$
k_{f}=\frac{1}{\rho_{0}} \log \left(\frac{1}{\tau-\varepsilon_{r}}\right) .
$$


Thus, even though the initial roundoff error in (2.7) is amplified exponentially, it only influences the solution in the lowest modes $|k| \leq k_{f}$.

Now compare the error $(v-u)$ and $(w-u)$ made in the two approximations. For the perturbed problem without filtering, the dominant contribution to $(v-u)$ is due to the growth of the largest wavenumber, $k_{\max }=N / 2$, so that

$$
|v-u| \approx \varepsilon_{r} e^{N t / 2}
$$

The approximation fails when this error is of size $O(1)$, which occurs when $t=T_{1}=$ $2 \log \left(1 / \varepsilon_{r}\right) N^{-1}$, a time that depends on the discretization and roundoff level, rather than on the singularity time of the continuous problem. Therefore, if $N \gg 1$, then $T_{1} \ll T_{0}$; i.e., the solution with roundoff error but no filtering diverges from the unperturbed solution well before the singularity time. One can see that by taking the roundoff error $\varepsilon_{r}$ to be exponentially small in $N$, this problem can be overcome. However, without the use of special software packages, this is impossible to do in real computations.

On the other hand, for the problem with filtering there are two sources of error. For $k \leq k_{f}$, the dominant error is due to the roundoff error at the wavenumber $k_{f}$; that is,

$$
|w-u| \approx \varepsilon_{r} e^{k_{f} t}=\frac{\varepsilon_{r}}{\left(\tau-\varepsilon_{r}\right)^{t / \rho_{0}}} .
$$

This error remains small, i.e., $O\left(\varepsilon_{r} / \tau\right)$, when $t=T_{0}=\rho_{0}$, which is the singularity time for the original problem. For $|k|>k_{f}$, the dominant contribution to the error comes from the smallest wavenumber $k_{f}+1$ that is set to zero, i.e.,

$$
|w-u| \approx e^{-\left(k_{f}+1\right)\left(\rho_{0}-t\right)}=\left(\tau-\varepsilon_{r}\right)^{1-t / \rho_{0}} e^{-\left(\rho_{0}-t\right)}=O\left(\tau^{1-t / \rho_{0}}\right) .
$$

This error becomes $O(1)$ when $t=T_{0}$. Putting these together, we see that $|w-u|$ is dominated by the error for $|k|>k_{f}$, and is $O\left(\tau^{1-t / \rho_{0}}\right)$.

These estimates for the errors show that the unfiltered problem with roundoff is close to the exact problem for only a short time, whereas the filtered problem is accurate almost up to the singularity time. This is precisely the behavior that has been observed in numerical simulation of the vortex sheet problem with and without filtering [15, 22].

Now, consider the following nonlinear modification of (2.2) Suppose that $\varepsilon$ is a small parameter, and take

$$
\left\{\begin{array}{l}
\eta_{t}=\mathcal{H}\left[\eta_{\alpha}\right]+\varepsilon A[\eta](\alpha, t) \\
A[\eta]=\frac{1}{\pi} \int_{-\infty}^{\infty} \frac{\left(\eta(\alpha)-\eta\left(\alpha^{\prime}\right)\right)^{2}}{\left(\alpha-\alpha^{\prime}\right)^{3}} d \alpha^{\prime} \\
\eta_{k}(0)=e^{-\rho_{0}|k|}
\end{array}\right.
$$

The choice of $A[\eta]$ is motivated by the expansion of the integral operator in the vortex sheet problem given in Section 5 for the discrete case (see (5.8)). The parameter $\varepsilon$ arises from rescaling the equation given small initial data. Although we cannot write the explicit solution to (2.12), we expect that its solution remains smooth until $t \approx \rho_{0}$, since $\varepsilon$ may be expected to keep the nonlinearity small. Roughly speaking, the operator $A[\eta]$ behaves like the product $\mathcal{H}\left[\eta_{\alpha}\right] \cdot \mathcal{H}\left[\eta_{\alpha}\right]$ (also see Section 5 ). Therefore the nonlinearity contains derivatives of the same order as the linear term. This fact, combined with the linear ill-posedness of the equation and the nonlocal nature of the nonlinear terms, requires the use of the abstract Cauchy-Kowalewski theorem to prove existence. The abstract Cauchy-Kowalewski theorem is a fundamental 
theorem on the existence of "analytic" solutions of functional differential equations, such as certain integro-differential equations. Actually, solutions are obtained in certain more general Banach spaces, but we always use analytic function spaces in this paper. For completeness, a precise statement of the continuous version of the abstract theorem is given in Appendix 1.

To prove that solutions to (2.12) exist up to $t \approx \rho_{0}$, it is instructive to rewrite (2.12) by integrating in time and using the linear integrating factor. This gives

$$
\eta(\alpha, t)=u(\alpha, t)+\varepsilon \int_{0}^{t} \widetilde{A}[\eta]\left(\alpha, t, t^{\prime}\right) d t^{\prime},
$$

where

$$
\hat{\tilde{A}}[\eta]\left(k, t, t^{\prime}\right)=e^{|k|\left(t-t^{\prime}\right)} \widehat{A}[\eta]\left(k, t^{\prime}\right)
$$

and $u$ is the solution to the linear equation (2.2). Thus, the linear part of the equation is integrated exactly. The abstract Cauchy-Kowalewski theorem can then be used to show existence of solutions to (2.13) for times arbitrarily close to $\rho_{0}$ for $\varepsilon$ close to 0 . We will use a discrete analogue of this exact integration of the linear part of the numerical scheme when we prove the convergence of the point vortex method for long times in Section 5.

Now, consider the case with both filtering and roundoff error. Since the equation (2.12) is nonlinear, the mode interaction makes the analysis of the effects of filtering and roundoff error in the equation (scheme) much more difficult than the case where filtering and roundoff error perturb only the initial condition. Therefore, we consider equation (2.12) with filtering and roundoff error in both the initial condition and the equation

$$
\begin{aligned}
\zeta_{t} & =P\left\{\mathcal{H}\left[\zeta_{\alpha}\right]+\varepsilon A[\zeta]+e_{r}\right\}, \\
\hat{\zeta}_{k}(0) & = \begin{cases}e^{-\rho_{0}|k|}+\varepsilon_{r} & \text { if } e^{-|k| \rho_{0}}+\varepsilon_{r} \leq \tau, \\
0 & \text { otherwise. }\end{cases}
\end{aligned}
$$

Again, we expect that if the roundoff error $e_{r}$ is small (i.e., $\left|\hat{e}_{r}(k)\right| \leq \varepsilon_{r}$ ), then solutions to (2.15) exist for $t \approx \rho_{0}$ as well. The presence of the nonlinear filtering and roundoff error makes it difficult to apply the abstract Cauchy-Kowalewski theorem directly to obtain this result. This is because additional assumptions are required to control the effects of the filtering and roundoff error. Using the appropriate assumptions, a careful mode by mode analysis shows that (2.15) does in fact have solutions existing for $t$ arbitrarily close to $\rho_{0}$ when $\varepsilon, \varepsilon_{r}$ are close to 0 . More specifically, it is shown in Sections 4 and 6 how (on the discrete level) the assumptions of the abstract Cauchy-Kowalewski theorem, its statement, and its proof must be modified to accomodate numerical filtering and roundoff error. The continuous version follows analogously.

Finally, the difference $\eta-\zeta$ can also be analyzed using the Cauchy-Kowalewski theorem as follows. Let $\nu=\eta-\zeta$; then

$$
\begin{aligned}
\nu(\alpha, t)= & u(\alpha, t)-w(\alpha, t)+F(\alpha, t) \\
& +\varepsilon \int_{0}^{t}\left(\widetilde{A}[\eta]\left(\alpha, t, t^{\prime}\right)-\widetilde{A}[\eta-\nu]\left(\alpha, t, t^{\prime}\right)\right) d t^{\prime},
\end{aligned}
$$




$$
\begin{aligned}
\widehat{F}(k, t) & =\int_{0}^{t} e^{|k|\left(t-t^{\prime}\right)} \hat{f}\left(k, t^{\prime}\right) d t^{\prime}, \\
f(\alpha, t) & =\mathcal{H}\left[\zeta_{\alpha}\right]+\varepsilon A[\zeta]-P\left\{\mathcal{H}\left[\zeta_{\alpha}\right]+\varepsilon A[\zeta]+e_{r}\right\},
\end{aligned}
$$

where $w$ is the solution of (2.6). Therefore, treat $F$ as a forcing function by using the fact that the solution $\zeta$ of (2.15) is smooth, appropriate properties of the nonlinear operator $A$, the consistency of the nonlinear filtering operator $P$, and the fact that $|u-w| \approx \tau^{1-t / \rho_{0}}$ is smooth; then the abstract Cauchy-Kowalewski theorem can be applied to show that smooth solutions to (2.16) exist in a slightly smaller time interval than for either $\eta$ or $\zeta$, but that this solution is, roughly speaking, of size $\tau^{1-t / \rho_{0}}\left(1+\varepsilon_{r} / \tau\right)$. This result is almost optimal, because it shows that the error $\eta-\zeta$ remains small for $t<\rho_{0}$, nearly the singularity time of the smooth solution.

The above scenario provides an outline for the approach we take to prove the convergence of the point vortex method in the following sections of this paper.

\section{VORTEX SHEETS AND MAIN RESULT}

The equation governing the motion of a periodic, planar vortex sheet, with singlesigned vortex sheet strength, is called the Birkhoff-Rott equation and is given by

$$
\begin{aligned}
\frac{d z^{*}}{d t} & =\frac{1}{4 \pi i} \mathrm{PV} \int_{-\pi}^{+\pi} \cot \left(\frac{z(\alpha, t)-z\left(\alpha^{\prime}, t\right)}{2}\right) d \alpha^{\prime}, \\
z(\alpha, 0) & =\alpha+s_{0}(\alpha),
\end{aligned}
$$

in which $z(\alpha, t)$ is the complex position of the interface and $\alpha$ is the Lagrangian circulation variable. If the initial vortex sheet strength is not single-signed, then the circulation variable cannot be used to parametrize the sheet, and the vortex sheet strength must be explicitly introduced. Our analysis also applies to this case; however we omit it here for simplicity. The explicit inclusion of the vortex sheet strength only introduces minor modifications of the analysis presented here, since the vortex sheet strength is time independent in the Lagrangian frame. See [16] for details.

In (3.1) the integral is a Cauchy principal value integral, due to the singularity at $\alpha^{\prime}=\alpha$, and ${ }^{*}$ denotes the complex conjugate. The periodicity implies that

$$
z(\alpha, t)=\alpha+s(\alpha, t)
$$

in which $s(\alpha, t)$ is $2 \pi$-periodic in $\alpha$ for each $t$. Since filtering can be applied only to functions that are periodic, the operator $P$ will be applied to $s$, but cannot be directly applied to $z$. For simplicity of notation, however, we denote

$$
P z=\alpha+P s .
$$

Denote by $\tilde{z}_{j}$ the discrete approximation of $z\left(\alpha_{j}, t\right)$, in which $\alpha_{j}=j h=2 \pi j / N$. Discretizing in space, leaving time continuous and applying the complex conjugation 
* yields the following semi-discrete versions of the PVM and MPVM:

$$
\begin{gathered}
\frac{d \tilde{z}_{j}}{d t}=-\frac{h}{4 \pi i} \sum_{\substack{l=-N / 2+1 \\
l \neq j}}^{N / 2} \cot \left(\frac{\tilde{z}_{j}-\tilde{z}_{l}}{2}\right)^{*} \quad(\mathrm{PVM}), \\
\frac{d \tilde{z}_{j}}{d t}=-\frac{h}{2 \pi i} \sum_{\substack{l=-N / 2+1 \\
l+j \text { odd }}}^{N / 2} \cot \left(\frac{\tilde{z}_{j}-\tilde{z}_{l}}{2}\right)^{*}(\mathrm{MPVM}) .
\end{gathered}
$$

Time discrete versions are obtained by applying any consistent time discretization. For simplicity, we consider the Euler time discretization. Including roundoff error and filtering, the fully discrete versions of (3.5) and (3.6) we consider are given by

$$
\begin{aligned}
& \tilde{z}_{j}^{n+1}=P\left\{\tilde{z}^{n}-\Delta t \frac{h}{4 \pi i} \sum_{\substack{l=-N / 2+1 \\
l \neq j}}^{N / 2} \cot \left(\frac{\tilde{z}_{j}-\tilde{z}_{l}}{2}\right)^{*}+\Delta t \cdot e_{r}\right\}_{j}(\mathrm{PVM}), \\
& \tilde{z}_{j}^{n+1}=P\left\{\tilde{z}^{n}-\Delta t \frac{h}{2 \pi i} \sum_{\substack{l=-N / 2+1 \\
l+j \text { odd }}}^{N / 2} \cot \left(\frac{\tilde{z}_{j}-\tilde{z}_{l}}{2}\right)^{*}+\Delta t \cdot e_{r}\right\}_{j} \quad(\mathrm{MPVM}),
\end{aligned}
$$

where $\tilde{z}_{j}^{n}$ is the discrete approximation to $z\left(\alpha_{j}, t_{n}\right)$ with $t_{n}=n \Delta t$. The roundoff error $e_{r}$ is assumed only to satisfy the bound

$$
\left|\hat{e}_{r}(k)\right| \leq \varepsilon_{r}
$$

uniformly in $k$, and is otherwise arbitrary. We refer the reader to $[15,22,8,14]$ for additional details.

We now introduce some notation. For $\rho>0$ and $f=f(\alpha)$, define a norm as follows:

$$
\|f\|_{\rho}=\sum_{k=-\infty}^{+\infty}\left|\hat{f}_{k}\right| e^{\rho|k|}
$$

where $\hat{f}_{k}$ is the $k$-th continuous Fourier coefficient of $f$. Assuming that $\|f\|_{\rho}$ is finite is roughly equivalent to assuming that $f(\alpha)$ is analytic in the strip $|\operatorname{Im}(\alpha)|<\rho$. Denote such analytic function spaces by $B_{\rho}$, i.e.,

$$
B_{\rho}=\left\{f:\|f\|_{\rho}<\infty\right\} .
$$

This norm will only be used in Theorem 3.1 below. All other norms in this paper are the discrete version of (3.9) given below.

Suppose now that $f$ is defined on the grid $\left\{\alpha_{j}=2 \pi j / N\right\}$ for $j=-N / 2+$ $1, \ldots, N / 2$. Then the discrete norm corresponding to (3.9) is given by

$$
\|f\|_{\rho}=\sum_{k=-N / 2+1}^{N / 2}\left|\hat{f}_{k}\right| e^{\rho|k|},
$$

where $\hat{f}_{k}$ is the $k$-th discrete Fourier coefficient of $f$. This is the norm in which convergence is proven.

In the continuous case, Caflisch and Orellana [5] showed the following nearequilibrium result. 
Theorem 3.1 (Long Time Existence; Caflisch and Orellana). Let $\epsilon$ be sufficiently small and $z(\alpha, 0)=\alpha+s_{0}(\alpha)$, with

$$
\left\|\partial_{\alpha} s_{0}\right\|_{\rho_{0}} \leq \epsilon \quad \text { and } \quad \hat{s}_{0}(0)=0 .
$$

Then, there exists $\kappa>1$ such that for $0 \leq t \leq T_{0}=2 \rho_{0} / \kappa$, the Birkhoff-Rott equation has an analytic solution $z(\alpha, t)=\alpha+s(\alpha, t)$ in which the perturbation $s$ continues to have 0 mean and remains of size $\epsilon$, i.e.,

$$
\left\|\partial_{\alpha} s(t, \alpha)\right\|_{\bar{\rho}(t)} \leq \epsilon \quad \text { and } \quad \hat{s}(0, t)=0,
$$

where $\bar{\rho}(t)=\rho_{0}-\kappa t / 2$, and, moreover, $\kappa$ is arbitrarily close to 1 when $\epsilon$ is close to 0 .

For initial data in $B_{\rho_{0}}$, there may be a singularity at position $\alpha_{*}$ in the complex $\alpha$ plane with $\rho_{*}=\left|\operatorname{Im}\left(\alpha_{*}\right)\right|>\rho_{0}$. For such data, linear theory predicts that a singularity will occur at time $t_{*}=2 \rho_{*}$. It was shown in [6] that, for $\varepsilon$ small and for a restricted set of initial data, the nonlinear and linear solutions are nearly identical up to and including the singularity time. Therefore, the time of existence $T_{0}$ is nearly optimal.

The main result of this paper is to show that, with roundoff error and filtering, both the PVM and the MPVM converge to the types of solutions considered by Caflisch and Orellana for a time interval almost up to the singularity time.

Theorem 3.2 (Almost Optimal Convergence with Roundoff Error and Filtering). Assume that $z(\alpha, t)=\alpha+s(\alpha, t)$ is a near equilibrium, periodic solution of the Birkhoff-Rott equation satisfying (3.12) and (3.13). Suppose that $\tilde{z}_{j}^{n}$ is the solution to either the PVM (3.7) or the MPVM (3.8) with simulated roundoff error and filtering. Then, for any $0<\omega<1$ there exist constants $C$ and $c$, independent of the numerical parameters but depending on $\omega$ and $z(\alpha, t)$, such that

$$
\begin{aligned}
& \left\|z^{n}-\tilde{z}^{n}\right\|_{\rho_{2}\left(t_{n}\right)} \leq C\left[\Delta t+h+\frac{\tau^{1-\omega}}{\Delta t}+\frac{\varepsilon_{r}}{\tau}\right] \quad(\mathrm{PVM}), \\
& \left\|z^{n}-\tilde{z}^{n}\right\|_{\rho_{2}\left(t_{n}\right)} \leq C\left[\Delta t+e^{-(1-\omega) \rho_{0} / h}+\frac{\tau^{1-\omega}}{\Delta t}+\frac{\varepsilon_{r}}{\tau}\right] \quad(\mathrm{MPVM}),
\end{aligned}
$$

for a time interval $0 \leq t_{n} \leq T_{2}$, where $t_{n}=n \Delta t$ and in which

$$
\begin{aligned}
T_{2} & =2 \omega \rho_{0} /(1+c \sqrt{\varepsilon}), \\
\rho_{2}(t) & =\omega \rho_{0}-(1+c \sqrt{\varepsilon}) t / 2,
\end{aligned}
$$

for $\varepsilon, \Delta t, h, \tau^{1-\omega} / \Delta t$, and $\varepsilon_{r} / \tau$ sufficiently small. Further, $2 \Delta t / h \leq 1$.

Remarks. 1. Theorem 3.2 shows that the numerical solutions $\tilde{z}_{j}^{n}$ converge to the true solution $z\left(\alpha_{j}, t_{n}\right)$ nearly up to the singularity time if the filter level $\tau$ and roundoff level $\varepsilon_{r}$ are treated as convergence parameters, in addition to the usual parameters $h$ and $\Delta t$. Note that since the MPVM is spectrally accurate [22], its spatial error $O\left(e^{-(1-\omega) \rho_{0} / h}\right)$ is much smaller than that for the PVM, which is $O(h)$. The convergence proofs for the PVM and MPVM differ only in the details of the estimates, such as the consistency estimate described above and the estimate on the linear part of the operator. Otherwise, the proofs are identical. The differences are pointed out in the proof as they appear.

2. Although the restriction $2 \Delta t / h \leq 1$ appears to be a Courant-Friedrichs-Lewy condition, it actually arises in the analysis of the filtering error and is probably not 
optimal. For example, in the proof we require that the derivative of the roundoff error satisfies $\left|k \hat{e}_{r}(k)\right| \leq \tau /(2 \Delta t)$. Since we assume that $\left|\hat{e}_{r}(k)\right| \leq \varepsilon_{r}$, the condition $2 \Delta t / h \leq 1$ naturally arises since $\varepsilon_{r}<\tau$.

3. One could also include roundoff error in the computation of the velocity. Consider, for example, the following version of the PVM:

$$
\tilde{z}_{j}^{n+1}=P\left\{\tilde{z}^{n}-\Delta t \frac{h}{4 \pi i} \sum_{\substack{l=-N / 2+1 \\ l \neq j}}^{N / 2} \cot \left(\frac{\tilde{z}_{j}-\tilde{z}_{l}+e_{r, j}-e_{r, l}}{2}\right)^{*}+\Delta t \cdot \tilde{e}_{r}\right\}_{j},
$$

where the functions $e_{r}$ and $\tilde{e}_{r}$ model the effects of roundoff error in the sheet positions and discrete velocity (cotangent sum). While this version of the PVM is appealing because it is perhaps more realistic than the method given in (3.7), it is significantly more difficult to analyze. Nevertheless, the techniques presented in this paper can be adapted to prove the convergence of (3.17), although much more restrictive, and hence less realistic, assumptions are required because of technical difficulties in the proof.

4. The proof of Theorem 3.2 relies on two versions of the discrete CauchyKowalewski theorem, which will be presented in the next section. One version includes the effects of numerical filtering and simulated roundoff error. In addition, careful estimates must be obtained for the filter $P$ and for the linear part of the discrete operator as well as the nonlinear part. The $\sqrt{\varepsilon}$ in the theorem arises naturally from the choice of constants in the application of the discrete CauchyKowalewski theorem.

5. If the solution is far from equilibrium, then the careful estimate on the nonlinear part of the discrete operator breaks down. It still can be estimated, however, but only in a way that results in short time convergence (if the true solution is smooth).

6. The technique of proof can be used to prove similar convergence theorems for other ill-posed problems to which the abstract Cauchy-Kowalewski theorem can be used to prove existence of analytic solutions in the continuous (spatially and temporally) case. Such problems include Rayleigh-Taylor unstable interfaces in inviscid, incompressible, and irrotational fluids as well as Saffman-Taylor unstable interfaces in a Hele-Shaw cell. See $[16,20,11,24,25,1]$, for example. The appropriate convergence proofs are then obtained by carefully analyzing the particular numerical method in question, obtaining an error equation, and then applying the discrete Cauchy-Kowalewski theorems to these cases. In order to be sure that the discrete Cauchy-Kowalewski theorem can be applied, two things are important. First, it must be possible to apply the continuous version to prove existence of analytic solutions. Second, it must be possible to write the spatial discretization so that it does not explicitly contain discrete derivatives of higher order than 1 . This is because the Cauchy-Kowalewski theorem applies only to first order operators. One consequence of this is that our proof cannot be directly applied to the case with surface tension (see $[10,2]$ for example), as this contains high order derivatives. However, the problem with non-zero surface tension is in fact linearly well-posed [2], and our analysis of Krasny filtering, presented here, combined with the convergence analysis presented in $[2,3]$, can be used to prove convergence in that case as well. Since surface tension regularizes the instability, one can always find a large 
but finite level of precision in which convergence in the presence of roundoff error may be proven (at a fixed time $T$ ) without Krasny filtering. For example, in the vortex sheet problem, one requires that the roundoff error $e_{r}$ satisfy a condition of the form $\left|\hat{e}_{r}(k)\right| \ll e^{-\rho(T) / \sigma}$, where $\sigma$ is the surface tension and $\rho(T)$ is the analyticity strip width at time $t=T$. In the Hele-Shaw problem, an analogous condition holds with $\sigma$ replaced by $\sqrt{\sigma}$. Thus, for small $\sigma$, these conditions may be difficult to satisfy, and so filtering or quadruple precision are typically used in the presence of small surface tension (e.g., [10]).

\section{The Discrete Cauchy-Kowalewski theorem}

The Cauchy-Kowalewski theorem is a fundamental theorem on the existence of analytic solutions to partial differential equations. In its abstract form [18, $19,21]$ it is applicable to integro-differential equation such as the Birkhoff-Rott equation (3.1). The abstract form of the theorem is directly applicable to semidiscrete equations (with continuous time), and needs only superficial modification for equations with discrete time. For completeness, a precise statement of the continuous time version is given in Appendix 1, although the proof of Theorem 3.2 only requires the discrete time versions given in this section.

Of course, for fully discrete equations the existence of solutions is trivial, and the real point of the theorem is to obtain uniform bounds on the solution. A discrete version of the theorem was proved in [8]. However, in that work the linear and nonlinear parts were not separated and so the result is not optimal. In this section, two versions of the discrete Cauchy-Kowalewski theorem are given. The first is a discrete version of the strengthened formulation and simplified proof of the abstract Cauchy-Kowalewski theorem by Safonov [21]. It has been modified to serve as a result for estimating perturbations, as needed for the nearly optimal convergence result with filtering. The second version modifies the first by allowing the inclusion of simulated roundoff error and numerical filtering. Again, a nearly optimal bound results. This is necessary for the convergence proof (presented in the next section) by providing uniform bounds on the numerical solutions of the PVM and MPVM with filtering and roundoff error.

Consider first the discrete equation without roundoff error and filtering

$$
\begin{aligned}
u_{n+1} & =L u_{n}+\Delta t A_{n}\left[u_{n}\right], \\
u_{0} & =0,
\end{aligned}
$$

in which $u_{n}=\left\{u_{n}^{i}\right\}$ is a discrete function in $B_{\rho}$. Suppose that the linear operator $L$ satisfies the following.

(i) $L$ is a linear operator on $B_{\rho}$, and there exists $\lambda_{0}$ such that for any $\rho^{\prime}>\rho>0$ and any $u \in B_{\rho+\lambda_{0} \Delta t}$

$$
\|L u\|_{\rho} \leq\|u\|_{\rho+\lambda_{0} \Delta t}
$$

and, for any $u \in B_{\rho^{\prime}}$,

$$
\|(L-I) u\|_{\rho} \leq \lambda_{0} \Delta t\left(\rho^{\prime}-\rho\right)^{-1}\|u\|_{\rho^{\prime}} .
$$

Suppose further that the nonlinear operator $A$ satisfies the following assumptions:

(ii) For any $0<\rho<\rho^{\prime}<\rho_{0}-\lambda_{0} n \Delta t, A_{n}$ is a continuous mapping of $\left\{u \in B_{\rho^{\prime}},\|u\|_{\rho^{\prime}} \leq R\right\}$ into $B_{\rho}$. 
(iii) For any $0<\rho<\rho^{\prime}<\rho_{0}-\lambda_{0} n \Delta t$, and for any $u, v \in B_{\rho^{\prime}}$ with $\|u\|_{\rho^{\prime}} \leq R$, $\|v\|_{\rho^{\prime}} \leq R$

$$
\left\|A_{n}[u]-A_{n}[v]\right\|_{\rho} \leq C_{1}\left(\rho^{\prime}-\rho\right)^{-1}\|u-v\|_{\rho^{\prime}},
$$

where $C_{1}$ is a constant independent of $u, v, \rho, \rho^{\prime}, n$. It may depend on $R$.

(iv) For any $0<\rho<\rho_{0}-\lambda_{0} n \Delta t$

$$
\left\|A_{n}[0]\right\|_{\rho} \leq K
$$

where $K$ is independent of $\rho$ and $n$.

(v) For any $0<\rho<\rho^{\prime}<\rho_{0}-\lambda_{0}(n+1) \Delta t$ and any $u \in B_{\rho^{\prime}}$ with $\|u\|_{\rho^{\prime}} \leq R$,

$$
\left\|A_{n+1}[u]-A_{n}[u]\right\|_{\rho} \leq C_{2}\left(\rho^{\prime}-\rho\right)^{-1} \Delta t,
$$

where $C_{2}$ is independent of $\rho, \rho^{\prime}, u, n$. It may depend on $R$ and boundedly on $\Delta t$ as $\Delta t \rightarrow 0$.

Theorem 4.1 (Discrete Cauchy-Kowalewski theorem). Suppose that L and $A$ satisfy assumptions (i)-(v) for some positive constants $\rho_{0}, \lambda_{0}, K, C_{1}, C_{2}$ and $R$. Then, there is a constant $\lambda$ (defined explicitly below) such that for $|n| \leq \rho_{0} /(\lambda \Delta t)$ the solution $u_{n}$ of equation (4.1) satisfies $u_{n} \in B_{\rho_{n}}$ and

$$
\left\|u_{n}\right\|_{\rho_{n}} \leq R
$$

in which $\rho_{n}=\rho_{0}-\lambda|n| \Delta t$ and $\lambda$ is given by

$$
\lambda=\max \left\{\begin{array}{l}
\lambda_{0}+\frac{R_{0} \rho_{0}^{1-\gamma}}{R(1-\gamma)}, \\
\lambda_{0}\left(1+\frac{R_{0} 2^{1+\gamma}}{R \gamma}\right), \\
\gamma^{-1}\left[C_{1} 2^{1+\gamma}\left(1+\frac{R}{R_{0}}\right)+2 C_{2} \frac{\rho_{0}^{\gamma}}{R_{0}}\right]
\end{array}\right.
$$

for any $0<\gamma<1$ and $R_{0} \geq K \rho_{0}^{\gamma}$.

The bound (4.7) will be used to estimate the difference between the solutions of the Birkhoff-Rott equation and the discretized equation, in order to show convergence of the discretized solutions. Note that in assumption (v), which does not appear in the statement of the continuous version, the values of the operator $A$ are compared at two different discrete time values $n$ and $n+1$. In the application to the convergence Theorem 3.2, the $n$ dependence of $A$ will be due to the time dependence of the exact solution. The proof of Theorem 4.1 is a simplified version of the proof of Theorem 4.2 and will not be presented separately.

The time interval of existence for the linear operator $L$ alone would be $\rho_{0} / \lambda_{0}$. If the nonlinear operator $A$ is small, as would be the case if the solution $u$ were small, then the constants $C_{1}, C_{2}, K$ and $R$ can be taken to be small. By careful choice of these constants, the resulting value of $\lambda$ will be only a small perturbation of $\lambda_{0}$; that is, by separating the linear and nonlinear parts of the equation, we obtain a nearly optimal time of existence.

Now consider the discrete equation with filtering and roundoff error

$$
\begin{aligned}
v_{n+1} & =P\left\{L v_{n}+\Delta t A_{n}\left[v_{n}\right]+\Delta t e_{r}\right\}, \\
v_{0} & =\text { given, }
\end{aligned}
$$

where $P$ is the nonlinear projection operator defined in (2.1) with filter level $\tau$ and $e_{r}$ is the simulated roundoff error which is assumed to satisfy the bound

(vi) $\left|\hat{e}_{r}(k)\right|<\varepsilon_{r}<\tau / 2 \Delta t$ for all wavenumbers $k$. 
In this theorem, the filter level $\tau$ is allowed to depend on the wavenumber $k$. This is needed in the convergence proof for the Birkhoff-Rott equation, since the CauchyKowalewski theorem will be applied to the derivative of the original equation.

The linear operator $L$, in addition to satisfying (i), is also assumed to be diagonalized by the Fourier transform, i.e.,

(vii) $\widehat{\operatorname{Lu}}(k)=l(k) \hat{u}(k)$.

The nonlinear operator $A_{n}$ is assumed to satisfy assumptions (ii)-(v). Unlike the previous case, non-zero initial data, $v_{0}$, is allowed. This is because the nonlinearity of $P$ makes it difficult to absorb the initial data into the equation. The projection is performed on the initial data, and it is further assumed to satisfy

(viii) $\left\|v_{0}\right\|_{\rho_{0}+\beta} \leq \delta R$ and $v_{0}=P v_{0}$, with $\delta<1$ and for some $\beta>0$.

Further, let $\Pi$ be an arbitrary linear Fourier projection operator such that $\widehat{\Pi u}(k)$ is either 0 or $\hat{u}(k)$, and set $0<\gamma<1$. Define the constants $R_{2}$ and $R_{1}$ such that

$$
\begin{aligned}
& R_{2} \geq \rho_{0}^{\gamma} \sup _{\Pi}\left\|A_{0}\left[\Pi v_{0}\right]\right\|_{\rho_{0}}, \\
& R_{1} \geq \frac{\rho_{0}^{\gamma}}{\Delta t}\left\|(L-I) v_{0}\right\|_{\rho_{0}} .
\end{aligned}
$$

Note that assumptions (i), (iii), (iv) and (viii) imply that $R_{2} \geq\left(\delta R C_{1} / \beta+K\right) \rho_{0}^{\gamma}$ and $R_{1} \geq \delta\left(\lambda_{0} R / \beta\right) \rho_{0}^{\gamma}$ satisfy (4.10) and (4.11).

Theorem 4.2. (Discrete Cauchy-Kowalewski Theorem with Roundoff Error and Filtering) Suppose that $P$ is defined by (2.1) and that $L, A, v_{0}, e_{r}$ and $\tau$ satisfy assumptions (i)-(viii) for some positive constants $\rho_{0}, \lambda_{0}, K, C_{1}, C_{2}, R, R_{2}, R_{1}, \delta, \beta$ and $\varepsilon_{r}$. Then, there is a constant $\lambda$ (defined explicitly below), such that for $n \leq$ $\rho_{0} /(\lambda \Delta t)$ the solution $v_{n}$ of equation (4.9) satisfies $v_{n} \in B_{\rho_{n}}$ and

$$
\left\|v_{n}\right\|_{\rho_{n}} \leq R
$$

in which $\rho_{n}=\rho_{0}-\lambda|n| \Delta t$ and $\lambda$ is given by

$$
\lambda=\max \left\{\begin{array}{l}
\lambda_{0}+\left(1+\frac{2 \varepsilon_{r} \Delta t}{\tau}\right)\left(1-\delta-\frac{\varepsilon_{r}}{\tau} t\right)^{-1} \frac{R_{2} \rho_{0}^{1-\gamma}}{R(1-\gamma)}, \\
\lambda_{0}\left[1+\frac{R_{2}}{R_{1} \gamma} 2^{1+\gamma}\left(1+\frac{2 \varepsilon_{r} \Delta t}{\tau}\right)\right]+\frac{\varepsilon_{r}}{\gamma \tau} \rho_{0}, \\
\frac{1}{\gamma}\left[C_{1} 2^{1+\gamma}\left(1+\frac{R_{1}}{R_{2}}+\frac{2 \varepsilon_{r} \Delta t}{\tau}\right)+2 C_{2} \frac{\rho_{0}^{\gamma}}{R_{2}}+\frac{2 \varepsilon_{r}}{\tau} \frac{R}{R_{2}} \rho_{0}^{\gamma}\right]
\end{array}\right.
$$

for any $0<\gamma<1$ and $t=\max (|n|) \Delta t$. If the filter level $\tau$ depends on $k$, then $\tau=\min \tau(k)$.

The bound (4.12) will be used to estimate the solution of the PVM and MPVM with filtering and roundoff error. This requires the additional assumptions (vi)(viii). Furthermore, if $\delta \ll 1$ (i.e., smallness of initial condition, see (viii)) and if $\varepsilon_{r} \ll \gamma \tau$, then $\lambda$ given by (4.13) is close to that given by (4.8), which gives a nearly optimal result in the case of filtering and roundoff error.

Before giving the proof of Theorem 4.2, we first prove the convergence result stated in Theorem 3.2. The proof of Theorem 4.2 will be given in Section 6 .

\section{Convergence proof}

In this section, the proof of the convergence theorem (Theorem 3.2) is presented. We begin by using the discrete Cauchy-Kowalewski theorem (Theorem 4.2) to prove uniform bounds for the numerical solution of the point vortex method with roundoff error and filtering. This bound plays an important role in the convergence proof 
by providing a control on the error introduced by the filtering. We treat the PVM and MPVM in tandem, pointing out their differences when necessary.

Define the discrete periodic function $\tilde{s}_{j}$ by $\tilde{s}_{j}=\tilde{z}_{j}-\alpha_{j}$, where $\alpha_{j}=j h$. Then, in terms of $\tilde{s}_{j}$, the PVM and MPVM with roundoff error and filtering are

$$
\tilde{s}_{j}^{n+1}=P\left\{\tilde{s}^{n}-\Delta t \frac{h}{4 \pi i} \sum_{\substack{l=-N / 2+1 \\ l \neq j}}^{N / 2} \cot \left(\frac{\alpha_{j}-\alpha_{l}+\tilde{s}_{j}-\tilde{s}_{l}}{2}\right)^{*}+\Delta t \cdot e_{r}\right\}_{j}
$$

$$
\tilde{s}_{j}^{n+1}=P\left\{\tilde{s}^{n}-\Delta t \frac{h}{2 \pi i} \sum_{\substack{l=-N / 2+1 \\ l+j \text { odd }}}^{N / 2} \cot \left(\frac{\alpha_{j}-\alpha_{l}+\tilde{s}_{j}-\tilde{s}_{l}}{2}\right)^{*}+\Delta t \cdot e_{r}\right\}_{j}
$$

It is convenient to expand the cotangent kernel as follows. Extend the discrete solution $\tilde{s}_{j}$ periodically outside the interval $(-N / 2+1 \leq j \leq N / 2)$, i.e., $\tilde{s}_{j+N}=\tilde{s}_{j}$. Then, it is a straightforward computation to see that, in terms of $\tilde{z}_{j}=\alpha_{j}+\tilde{s}_{j}$ so extended, one gets

$$
\frac{h}{4 \pi i} \sum_{\substack{l=-N / 2+1 \\ l \neq j}}^{N / 2} \cot \left(\frac{\tilde{z}_{j}-\tilde{z}_{l}}{2}\right)=\lim _{M \rightarrow \infty} \frac{h}{2 \pi i} \sum_{\substack{l=-N(M+1 / 2)+1 \\ l \neq j}}^{N(M+1 / 2)} \frac{1}{\tilde{z}_{j}-\tilde{z}_{l}}
$$

for the PVM (see [4] for details). We adopt the notation

$$
F^{\mathrm{PVM}}[\tilde{s}]_{j} \equiv \frac{h}{2 \pi i} \sum_{l \neq j} \frac{1}{\tilde{z}_{j}-\tilde{z}_{l}} \equiv \lim _{M \rightarrow \infty} \frac{h}{2 \pi i} \sum_{\substack{l=-N(M+1 / 2)+1 \\ l \neq j}}^{N(M+1 / 2)} \frac{1}{\tilde{z}_{j}-\tilde{z}_{l}} .
$$

The operator $F^{\mathrm{MPVM}}$ is analogously defined.

Define $D$ to be the discrete spectral derivative, i.e., $\widehat{D}=i k$ for $-N / 2+1 \leq$ $k \leq N / 2$ and periodically extended to all $k$. Then, it was shown in [8] that if $\|D f\|_{\rho^{\prime}} \leq 1 / 2$ and $\|D g\|_{\rho^{\prime}} \leq 1 / 2$, where $\|\cdot\|_{\rho}$ is the discrete norm in (3.11), then

$$
\|D F[f]-D F[g]\|_{\rho} \leq \frac{c}{\rho^{\prime}-\rho}\|D f-D g\|_{\rho^{\prime}}
$$

where $F$ is either $F^{\mathrm{PVM}}$ or $F^{\mathrm{MPVM}}$.

We now separate the operators $F^{\mathrm{PVM}}$ and $F^{\mathrm{MPVM}}$ into their linear and nonlinear parts. Write

$$
F^{\mathrm{PVM}}=F_{\mathrm{L}}^{\mathrm{PVM}}+F_{\mathrm{NL}}^{\mathrm{PVM}} \text { and } \quad F^{\mathrm{MPVM}}=F_{\mathrm{L}}^{\mathrm{MPVM}}+F_{\mathrm{NL}}^{\mathrm{MPVM}},
$$

where

$$
F_{\mathrm{L}}^{\mathrm{PVM}}[\tilde{s}]_{j}=\frac{h}{2 \pi i} \sum_{l \neq j} \frac{\tilde{s}_{j}-\tilde{s}_{l}}{\left(\alpha_{j}-\alpha_{l}\right)^{2}} \quad \text { and } \quad F_{\mathrm{NL}}^{\mathrm{PVM}}=F^{\mathrm{PVM}}-F_{\mathrm{L}}^{\mathrm{PVM}} \text {, }
$$

and analogously for the MPVM. 
If $\|D \tilde{s}\|_{\rho}<1$, then $F_{\mathrm{NL}}^{\mathrm{PVM}}$ can be expanded in the series [8]

$$
F_{\mathrm{NL}}^{\mathrm{PVM}}[\tilde{s}]_{j}=\frac{h}{2 \pi i} \sum_{l \neq j} \sum_{m=2}^{\infty} \frac{\left(\tilde{s}_{j}-\tilde{s}_{l}\right)^{m}}{\left(\alpha_{j}-\alpha_{l}\right)^{m+1}} .
$$

The operator $F_{\mathrm{NL}}^{\mathrm{MPVM}}$ has an analogous expansion.

Discrete Fourier analysis can be used to analyze both the linear and the nonlinear parts of $F^{\mathrm{PVM}}$ and $F^{\mathrm{MPVM}}$. In fact, $F_{\mathrm{L}}^{\mathrm{PVM}}$ is exactly $1 / 2 i$ times the trapezoidal quadrature (omitting the singular point) of the continuous spatial derivative of the Hilbert transform

$$
\mathcal{H}\left[f_{\alpha}\right](\alpha)=\frac{1}{\pi} \mathrm{P} . \mathrm{V} \cdot \int_{-\infty}^{\infty} \frac{f(\alpha)-f\left(\alpha^{\prime}\right)}{\left(\alpha-\alpha^{\prime}\right)^{2}} d \alpha^{\prime}
$$

applied to periodic functions (see [13]). It is not difficult to see that

$$
\widehat{F}_{\mathrm{L}}^{\mathrm{PVM}}=\frac{1}{2 i}|k|\left(1-\frac{|k|}{N}\right)
$$

and

$$
\widehat{F}_{\mathrm{L}}^{\mathrm{MPVM}}=\frac{1}{2 i}|k|
$$

for $-N / 2+1 \leq k \leq N / 2$ and periodically extended to all $k$. See [4] for example. This should be contrasted with the continuous case $\left(\frac{1}{2 i} H \partial_{\alpha}\right)$, which has the symbol $\frac{1}{2 i}|k|$ for all $k$.

We now drop the PVM, MPVM notation and simply refer to $F_{\mathrm{L}}$ and $F_{\mathrm{NL}}$. Both the PVM and MPVM schemes can be written as

$$
\hat{s}_{j}^{n+1}=P\left\{\tilde{s}_{j}^{n}+\Delta t F_{\mathrm{L}}\left[\tilde{s}^{n}\right]^{*}+\Delta t F_{\mathrm{NL}}\left[\tilde{s}^{n}\right]^{*}+\Delta t e_{r}\right\}_{j} .
$$

Define

$$
\begin{aligned}
v^{n+1} & =D \tilde{s}^{n+1}, \\
L v^{n} & =v^{n}+\Delta t F_{\mathrm{L}}\left[v^{n}\right]^{*}, \\
\mathcal{A}\left[v^{n}\right] & =D F_{\mathrm{NL}}\left[D^{-1} v^{n}\right]^{*}=D F_{\mathrm{NL}}\left[\tilde{s}^{n}\right]^{*}, \\
\tilde{e}_{r} & =D e_{r}^{*} .
\end{aligned}
$$

Taking a discrete derivative $D$, i.e., $\widehat{D}=i k$, of (5.12) yields

$$
v^{n+1}=\widetilde{P}\left\{L v^{n}+\Delta t \mathcal{A}\left[v^{n}\right]+\Delta t \tilde{e}_{r}\right\},
$$

where $\widetilde{P}$ denotes the projection operator with $\tau$ replaced by $\tau|k|$ for each Fourier mode $-N / 2+1 \leq k \leq N / 2$. The reason for this is that $D$ has been passed through the original projection $P$. This makes the equations quasilinear.

Equation (5.17) is exactly in the form required to apply Theorem 4.2. It remains now to verify the assumptions (i)-(viii).

(1) Since $\left|\widehat{F}_{\mathrm{L}}\right| \leq|k| / 2$, for both the PVM and MPVM, we have

$$
\begin{gathered}
\|L v\|_{\rho}=\left\|\left(I+\Delta t F_{\mathrm{L}}^{*}\right)[v]\right\|_{\rho} \leq\|v\|_{\rho+\Delta t / 2}, \\
\|(L-I) v\|_{\rho}=\Delta t\left\|F_{\mathrm{L}}[v]^{*}\right\|_{\rho} \leq \frac{\Delta t}{2}\left(\rho^{\prime}-\rho\right)^{-1}\|v\|_{\rho^{\prime}},
\end{gathered}
$$

with $\rho^{\prime}>\rho>0$ and $v \in B_{\rho+\Delta t / 2}, B_{\rho^{\prime}}$, as required. This shows that (i) is satisfied with $\lambda_{0}=1 / 2$. 
(2) We have $\|\mathcal{A}[u]-\mathcal{A}[v]\|_{\rho}=\left\|D F_{\mathrm{NL}}\left[D^{-1} u\right]^{*}-D F_{\mathrm{NL}}\left[D^{-1} v\right]^{*}\right\|_{\rho}$. In [8], it was shown that for the PVM if $\|u\|_{\rho^{\prime}},\|v\|_{\rho^{\prime}} \leq R<1 / 2$ then there exists a $c>0$ such that

$$
\left\|D F_{\mathrm{NL}}\left[D^{-1} u\right]^{*}-D F_{\mathrm{NL}}\left[D^{-1} v\right]^{*}\right\|_{\rho} \leq \frac{c R}{\rho^{\prime}-\rho}\|u-v\|_{\rho^{\prime}}
$$

where $0<\rho<\rho^{\prime}$ and $c$ is independent of $u, v, \rho$ and $\rho^{\prime}$. The analysis in [8] can be straightforwardly extended to the case of the MPVM. This shows that (ii) and (iii) are satisfied with $C_{1}=c R$ and $R<1 / 2$.

(3) $\mathcal{A}[0]=0$, so that (iv) is satisfied with $K=0$.

(4) Since $\mathcal{A}$ is independent of $n,(\mathrm{v})$ is satisfied with $C_{2}=0$.

(5) Recall that we assumed that the roundoff error $\left|\hat{e}_{r}(k)\right| \leq \varepsilon_{r}$; this implies $\left|\hat{\tilde{e}}_{r}(k)\right| \leq \varepsilon_{r} / h$. So, if $\Delta t / h \leq 1 / 2$, then (vi) is satisfied.

(6) Condition (vii) is satisfied with $l(k)=\frac{1}{2 i}|k|\left(1-\frac{|k|}{N}\right)$ for the PVM and $l(k)=$ $\frac{1}{2 i}|k|$ for the MPVM, both appropriately extended.

(7) Now consider the initial condition. Suppose that $v^{0}=P v^{0}$. Further suppose that $\left\|v^{0}\right\|_{\rho_{0}+\beta_{0}} \leq \varepsilon$ for some $\beta_{0}>0$. This is essentially equivalent to assuming that $\left\|\partial_{\alpha} s_{0}\right\|_{\rho_{0}+\beta_{0}} \leq \varepsilon$. Then, (viii) is satisfied with $R=\varepsilon / \delta$ for any $0<\delta<1$.

We are now almost ready to apply Theorem 4.2. It remains to choose $R_{1}, R_{2}$ according to (4.10) and (4.11). This is a little delicate, as we want to ensure that the $\lambda=\lambda_{f}$ resulting from the theorem is a small perturbation of $\lambda_{0}=1 / 2$. One can check that for $\delta=1 / 2$ and $\gamma=1 / 2$, by choosing

$$
R_{1}=\frac{\varepsilon \rho_{0}^{1 / 2}}{2 \beta_{0}} \quad \text { and } \quad R_{2}=2^{7 / 2} \frac{c \varepsilon^{2} \rho_{0}^{1 / 2}}{\beta_{0}}
$$

and requiring that $\varepsilon, \varepsilon / \beta_{0}$ and $\varepsilon_{r}$ be small enough, Theorem 4.2 does indeed yield

$$
\left\|D \tilde{s}^{n}\right\|_{\rho_{n}} \leq 2 \varepsilon, \quad \text { which implies that }\left\|\tilde{s}^{n}\right\|_{\rho_{n}} \leq 2 \varepsilon,
$$

where

$$
\rho_{n}=\rho_{0}-\lambda_{f} n \Delta t
$$

and $\lambda_{f}$ is a small perturbation of the linear result $\lambda_{0}=1 / 2$; i.e.,

$$
\lambda_{f}=\frac{1}{2}+c \varepsilon,
$$

where $c$ is a global constant that can be bounded independently of all the numerical parameters. The bound (5.22) will be used to control the effect of the filtering error in the convergence of the numerical scheme.

Now we turn to the question of the convergence of the numerical scheme. Following the analysis in [8], it can be shown that, for either the PVM or MPVM, the continuous solution $s_{j}^{n}=z\left(\alpha_{j}, n \Delta t\right)-\alpha_{j}$ satisfies the discrete equation

$$
s_{j}^{n+1}=s_{j}^{n}+\Delta t\left(F_{\mathrm{L}}\left[s^{n}\right]_{j}^{*}+F_{\mathrm{NL}}\left[s^{n}\right]_{j}^{*}\right)+\Delta t\left(f_{\Delta t}^{n}+f_{h}^{n}\right)_{j},
$$

where $F_{\mathrm{L}}$ and $F_{\mathrm{NL}}$ are as defined before and $f_{\Delta t}^{n}, f_{h}^{n}$ are the temporal and spatial consistency errors, respectively. Assuming that $\bar{\rho}(t)=\rho_{0}-\kappa t / 2$ and $\left\|\partial_{\alpha} s(\alpha, t)\right\|_{\bar{\rho}(t)}$ $\leq \varepsilon$ as in Theorem 3.1, the analysis in [8] shows that with $\rho<\bar{\rho}(t)$, the temporal error is bounded by

$$
\left\|D f_{\Delta t}^{n}\right\|_{\rho} \leq \frac{c \varepsilon \Delta t}{(\bar{\rho}(t)-\rho)^{3}}
$$


for both the PVM and MPVM. The spatial error is bounded by

$$
\left\|D f_{h}^{n}\right\|_{\rho} \leq \frac{c \varepsilon}{(\bar{\rho}(t)-\rho)^{3}} \cdot \begin{cases}h, & \text { for PVM, } \\ e^{-\bar{\rho}(t) / h}, & \text { for MPVM } .\end{cases}
$$

The -3 power in the estimates (5.26) and (5.27) is not of much significance and is probably not optimal. The bound can be controlled by keeping $\rho$ sufficiently smaller then $\bar{\rho}$. For example, if $\rho<\omega \rho_{0}-\kappa t / 2$ with $0<\omega<1$, then

$$
\left\|D f_{\Delta t}^{n}\right\|_{\rho} \leq \frac{c \varepsilon \Delta t}{\left[(1-\omega) \rho_{0}\right]^{3}}
$$

and

$$
\left\|D f_{h}^{n}\right\|_{\rho} \leq \frac{c \varepsilon}{\left[(1-\omega) \rho_{0}\right]^{3}} \cdot \begin{cases}h, & \text { for PVM } \\ e^{-(1-\omega) \rho_{0} / h}, & \text { for MPVM }\end{cases}
$$

These can be made arbitrarily small, for any $\varepsilon$, by taking $\Delta t$ and $h$ sufficiently small with respect to $(1-\omega) \rho_{0}$ and $c \varepsilon$.

Define the error to be $d^{n}=s^{n}-\tilde{s}^{n}=z^{n}-\tilde{z}^{n}$. Letting $u^{n+1}=D d^{n+1}$ gives the error equation

$$
u^{n+1}=L u^{n}+\Delta t A_{n}\left[u^{n}\right],
$$

where $L$ is defined as in (5.14) and the nonlinear operator $A_{n}$ is given by

$$
A_{n}\left[u^{n}\right]=D f_{\mathrm{NL}}\left[s^{n}\right]^{*}-D F_{\mathrm{NL}}\left[s^{n}-D^{-1} u^{n}\right]^{*}+e_{\Delta t}^{n}+e_{h}^{n}+e_{f}^{n}
$$

and $e_{\Delta t}^{n}, e_{h}^{n}, e_{f}^{n}$ denote the temporal, spatial, and filtering errors, respectively. They are given by

$$
\begin{aligned}
e_{\Delta t}^{n} & =D f_{\Delta t}^{n}, \\
e_{h}^{n} & =D f_{h}^{n}, \\
e_{f}^{n} & =\frac{1}{\Delta t} D\left[L \tilde{s}^{n}+\Delta t F_{\mathrm{NL}}\left[\tilde{s}^{n}\right]-P\left\{L \tilde{s}^{n}+\Delta t F_{\mathrm{NL}}\left[\tilde{s}^{n}\right]+\Delta t e_{r}\right\}\right]^{*} .
\end{aligned}
$$

Consequently, equation (5.30) is exactly in the form to which Theorem 4.1 may be applied once conditions (i)-(v) are verified. This is what we do next.

(1) Equations (5.18) and (5.19) ensure that (i) is satisfied with $\lambda_{0}=\max \left(\lambda_{f}, \kappa / 2\right)$, since both $\lambda_{f}$ and $\kappa / 2$ are larger than $1 / 2$. We need $\lambda_{0} \geq \kappa / 2$ to control the discretization errors, as seen above, and we need $\lambda_{0} \geq \lambda_{f}$ to control the filtering error. This will be made apparent below when we verify (iv).

(2) Suppose that $0<\omega<1$ and $0<\rho<\rho^{\prime}<\omega \rho_{0}-\lambda_{0} n \Delta t$, with $\lambda_{0}$ as above. Suppose that $\|u\|_{\rho^{\prime}},\|v\|_{\rho^{\prime}} \leq R$ and $\left\|\partial_{\alpha} s^{n}\right\|_{\rho^{\prime}} \leq \varepsilon$ and $R \leq \varepsilon<1 / 2$. Then

$$
\begin{aligned}
\left\|A_{n}[u]-A_{n}[v]\right\|_{\rho} & =\left\|D F_{\mathrm{NL}}\left[s^{n}-D^{-1} u\right]^{*}-D F_{\mathrm{NL}}\left[s^{n}-D^{-1} v\right]^{*}\right\|_{\rho} \\
& \leq \frac{c \varepsilon}{\rho^{\prime}-\rho}\|u-v\|_{\rho^{\prime}} .
\end{aligned}
$$

This follows from the analysis in [8], and shows that (ii) and (iii) are satisfied with $C_{1}=c \varepsilon$ and $R \leq \varepsilon<1 / 2$.

(3) Consider $\left\|A_{n}[0]\right\|_{\rho}$. We have the estimate

$$
\left\|A_{n}[0]\right\|_{\rho} \leq\left\|e_{\Delta t}^{n}\right\|_{\rho}+\left\|e_{h}^{n}\right\|_{\rho}+\left\|e_{f}^{n}\right\|_{\rho} .
$$


For $0<\rho<\omega \rho_{0}-\lambda_{0} n \Delta t,(5.28)$ and (5.29) show that

$$
\left\|e_{\Delta t}^{n}\right\|_{\rho}+\left\|e_{h}^{n}\right\|_{\rho} \leq \frac{c \varepsilon}{\left[(1-\omega) \rho_{0}\right]^{3}} \cdot \begin{cases}\Delta t+h, & \text { for PVM, } \\ \Delta t+e^{-(1-\omega) \rho_{0} / h}, & \text { for MPVM }\end{cases}
$$

It now remains to estimate the filtering error $e_{f}^{n}$. For this, we use the following two lemmas, which show that $P$ is consistent both with and without roundoff error.

Lemma 5.1 (Consistency Property of $P$ ). Let $0<\rho<\rho^{\prime}$ and assume that $f \in$ $B_{\rho^{\prime}}$. Then

$$
\|(I-P) f\|_{\rho} \leq\|f\|_{\rho^{\prime}}^{\rho / \rho^{\prime}}\left(2+\rho^{-1}+\left(\rho^{\prime}-\rho\right)^{-1}\right) \tau^{1-\rho / \rho^{\prime}} .
$$

If $\tau=\tau(k)$, then $\tau=\max _{k}|\tau(k)|$ in (5.38).

Lemma 5.2 (Filtering with Roundoff Error). Let $0<\rho<\rho^{\prime}$ and assume that $f \in B_{\rho^{\prime}}$. Let $e_{r}$ represent simulated roundoff error, with $\left|\hat{e}_{r}(k)\right| \leq \varepsilon_{r}<\tau / 2$ for all $k$. Two estimates on the filtering in the presence of roundoff error are the following:

$$
\begin{aligned}
& \left\|P\left(f+e_{r}\right)-P f\right\|_{\rho} \leq \frac{\varepsilon_{r}}{\rho^{\prime}}\left(\frac{\|f\|_{\rho^{\prime}}}{2 \tau}\right)^{\rho / \rho^{\prime}} \log \left(\frac{\|f\|_{\rho^{\prime}}}{2 \tau}\right)+4\left\|f-P_{2 \tau} f\right\|_{\rho^{\prime}}, \\
& \left\|P\left(f+e_{r}\right)-P f\right\|_{\rho} \leq \frac{\varepsilon_{r}}{2 \tau}\left[1+\left(\rho^{\prime}-\rho\right)^{-1}\right]\|f\|_{\rho^{\prime}}+4\left\|f-P_{2 \tau} f\right\|_{\rho^{\prime}},
\end{aligned}
$$

in which $P_{2 \tau}$ is the filtering operator of (2.1) with $\tau$ replaced by $2 \tau$. If $\tau=\tau(k)$, then in the above $\tau=\min _{k}|\tau(k)|$, except in the projections where the dependence of $\tau$ on $k$ is kept.

A third lemma, which we present but do not actually use, shows the sense in which $P$ is a stable operator.

Lemma 5.3 (Stability Property of $P$ ). Let $0<\rho<\rho^{\prime}$ and assume that $f \in B_{\rho^{\prime}}$ and $g \in B_{\rho}$. Then

$$
\|P f-P g\|_{\rho} \leq\|f-g\|_{\rho}+\|f\|_{\rho^{\prime}}^{\rho / \rho^{\prime}}\left(2+\rho^{-1}+\left(\rho^{\prime}-\rho\right)^{-1}\right) \tau^{1-\rho / \rho^{\prime}} .
$$

If $\tau=\tau(k)$, then $\tau=\max _{k} \tau(k)$ in $(5.41)$.

The proofs of these three lemmas are given in Appendix 2.

Now, estimate $e_{f}^{n}$ by

$$
\begin{aligned}
\left\|e_{f}\right\|_{\rho} \leq & \frac{1}{\Delta t}\left(\rho^{\prime}-\rho\right)^{-1}\left\|(I-P)\left(L \tilde{s}^{n}+\Delta t F_{\mathrm{NL}}\left[\tilde{s}^{n}\right]\right)\right\|_{\rho^{\prime}} \\
& +\frac{1}{\Delta t}\left(\rho^{\prime}-\rho\right)^{-1}\left\|P\left(L \tilde{s}^{n}+\Delta t F_{\mathrm{NL}}\left[\tilde{s}^{n}\right]\right)-P\left(L \tilde{s}^{n}+\Delta t F_{\mathrm{NL}}\left[\tilde{s}^{n}\right]+\Delta t e_{r}\right)\right\|_{\rho^{\prime}} .
\end{aligned}
$$

As we have seen by applying Theorem 4.2 to the discrete filtered equation, $\tilde{s}^{n}$ satisfies the uniform bound

$$
\left\|\tilde{s}^{n}\right\|_{\tilde{\rho}} \leq 2 \varepsilon
$$

with $\tilde{\rho} \leq \rho_{0}-\lambda_{f} n \Delta t$, where $\lambda_{f}=1 / 2+c \varepsilon$. Then, by restricting $\rho$ and $\rho^{\prime}$ in (5.42) by $0<\rho<\rho^{\prime}<\omega \rho_{0}-\lambda_{f} n \Delta t$, Lemmas 5.1 and 5.2, together with the estimates (5.18)-(5.20), can be applied to show that

$$
\left\|e_{f}\right\|_{\rho} \leq c_{\omega} \varepsilon\left(\frac{\tau^{1-\omega}}{\Delta t}+\frac{\varepsilon_{r}}{\tau}\right)
$$


Putting everything together shows that (iv) is satisfied with

$$
\begin{aligned}
& K=c_{\omega} \varepsilon\left(\Delta t+h+\frac{\tau^{1-\omega}}{\Delta t}+\frac{\varepsilon_{r}}{\tau}\right) \quad(\mathrm{PVM}), \\
& K=c_{\omega} \varepsilon\left(\Delta t+e^{(1-\omega) \rho_{0} / h}+\frac{\tau^{1-\omega}}{\Delta t}+\frac{\varepsilon_{r}}{\tau}\right) \quad(\mathrm{MPVM}) .
\end{aligned}
$$

Again, $c_{\omega}$ is a generic constant depending only on $\omega$ and $z(\alpha, t)$.

(5) Finally, it remains to show that (v) is satisfied. We have

$$
\begin{aligned}
A_{n+1}[u]-A_{n}[u]= & D F_{\mathrm{NL}}\left[s^{n+1}\right]^{*}-D F_{\mathrm{NL}}\left[s^{n+1}-D^{-1} u\right]^{*} \\
& -D F_{\mathrm{NL}}\left[s^{n}\right]^{*}+D F_{\mathrm{NL}}\left[s^{n}-D^{-1} u\right]^{*} \\
& +\left(e_{\Delta t}^{n+1}-e_{\Delta t}^{n}\right)+\left(e_{h}^{n+1}-e_{h}^{n}\right)+\left(e_{f}^{n+1}-e_{f}^{n}\right) .
\end{aligned}
$$

It is not difficult to show that

$$
\left\|\left(e_{t}^{n+1}+e_{t}^{n}\right)+\left(e_{h}^{n+1}-e_{h}^{n}\right)+\left(e_{f}^{n+1}-e_{f}^{n}\right)\right\|_{\rho} \leq \Delta t K
$$

for $\rho \leq \omega \rho_{0}-\lambda_{0} \Delta t$, with $K$ defined as above but with a redefined constant $c_{\omega}$. The estimate of the remaining terms in (5.45) is more subtle.

The obvious estimate of these terms is

$$
\begin{aligned}
\| D F_{\mathrm{NL}}\left[s^{n+1}\right]- & D F_{\mathrm{NL}}\left[s^{n+1}-D^{-1} u\right]-D F_{\mathrm{NL}}\left[s^{n}\right]+D F_{\mathrm{NL}}\left[s^{n}-D^{-1} u\right] \|_{\rho} \\
\leq & \left\|D F_{\mathrm{NL}}\left[s^{n+1}\right]-D F_{\mathrm{NL}}\left[s^{n}\right]\right\|_{\rho} \\
& +\left\|D F_{\mathrm{NL}}\left[s^{n+1}-D^{-1} u\right]-D F_{\mathrm{NL}}\left[s^{n}-D^{-1} u\right]\right\|_{\rho} \\
\leq & \frac{c}{\rho^{\prime}-\rho}\left\|D s^{n+1}-D s^{n}\right\|_{\rho^{\prime}} \\
\leq & \frac{c \varepsilon \Delta t}{\rho^{\prime}-\rho}
\end{aligned}
$$

for $\rho<\rho^{\prime} \leq \omega \rho_{0}-\lambda_{0}(n+1) \Delta t$, provided that $\|u\|_{\rho^{\prime}} \leq R<1 / 2$. We have further used the inequality $\left\|D\left(s^{n+1}-s^{n}\right)\right\|_{\rho} \leq c \varepsilon \Delta t$, and $c$ is a generic constant. Combining (5.48) with (5.46) shows that

$$
\left\|A_{n+1}[u]-A_{n}[u]\right\|_{\rho} \leq \frac{c_{\omega} \Delta t}{\rho^{\prime}-\rho}\left[\varepsilon+\rho_{0} K\right] .
$$

This suggests that (v) is satisfied with $C_{2}=c_{\omega}\left[\varepsilon+\rho_{0} K\right]$. It turns out, however, that this estimate is not sufficient to obtain convergence. The reason is the following. When Theorem 4.1 is applied to the error equation (5.30), the result is that the error $\left\|u^{n+1}\right\|_{\rho} \leq R$ for $\rho<\omega \rho_{0}-\lambda(n+1) \Delta t$. To obtain convergence, it is necessary that $R \rightarrow 0$ as the numerical parameters $\rightarrow 0$. An optimal result is obtained by having $\lambda$ be a small perturbation of $\lambda_{0}$. The actual value of $\lambda$ is given in (4.8) of Theorem 4.1. Note that $\lambda$ depends on the quantities $\rho_{0}, \gamma, \lambda_{0}, R_{0} / R, R / R_{0}$ and $C_{2} / R_{0}$. An optimal $\lambda$ requires the ratios $R_{0} / R, R / R_{0}$ and $C_{2} / R_{0}$ to be small, even in the limit as the numerical parameters $\rightarrow 0$. Since $R \rightarrow 0$ in this limit, in order for the ratios to be well-defined, we must have $R_{0} \rightarrow 0$, which implies that $C_{2} \rightarrow 0$ in this limit as well. However, the value of $C_{2}$ we obtained above does not vanish in the limit, because the first term depends on $\varepsilon$, which is independent of the numerical parameters. Thus, an optimal result is not obtained using this value of $C_{2}$.

This difficulty arises because the terms involving $u$ and $s^{n+1}, s^{n}$ have been handled in (5.47) and (5.48) so that the dependence on $u$ (the error) is removed. It 
is exactly this dependence that is required for $C_{2} \rightarrow 0$. It turns out that if the estimate is not broken up as in (5.47), then the highest order terms involving $s^{n+1}$ and $s^{n}$ cancel and only cross terms involving $u$ and $s^{n+1}-s^{n}$ remain! This shows that $C_{2}$ in fact $\rightarrow 0$ as the numerical parameters vanish. The following lemma summarizes the result.

Lemma 5.4 (Time Difference of Nonlinear Operator). Let $\|u\|_{\rho} \leq R$ and $\|D f\|_{\rho}$, $\|D g\|_{\rho} \leq \widetilde{R}$ with $R \leq \widetilde{R}<1 / 3$. Then

$$
\left\|F_{\mathrm{NL}}[f]-F_{\mathrm{NL}}\left[f-D^{-1} u\right]-F_{\mathrm{NL}}[g]+F_{\mathrm{NL}}\left[g-D^{-1} u\right]\right\|_{\rho} \leq c R\|D(f-g)\|_{\rho} .
$$

The constant $c$ is independent of $f, g$ and $u$.

The proof uses discrete Fourier analysis to obtain the explicit cancellation, and will be presented in Appendix 3.

Applying Lemma 5.4 with $f=s^{n+1}$ and $g=s^{n}$ to (5.45) gives

$$
\left\|A_{n+1}[u]-A_{n}[u]\right\|_{\rho} \leq \frac{c}{\rho^{\prime}-\rho}\left[R\left\|D\left(s^{n+1}-s^{n}\right)\right\|_{\rho^{\prime}}+\Delta t \rho_{0} K\right],
$$

where $\rho<\rho^{\prime}<\omega \rho_{0}-\tilde{\lambda}(n+1) \Delta t,\|u\|_{\rho^{\prime}} \leq R$ and $\|D s\|_{\bar{\rho}(t)} \leq \varepsilon$. Using (5.25) and the analyticity of the smooth solution $s$, it is straightforward to show that [8]

$$
\left\|D\left(s^{n+1}-s^{n}\right)\right\|_{\rho^{\prime}} \leq c \varepsilon \Delta t .
$$

Using (5.52) and (5.51) gives

$$
\left\|A_{n+1}[u]-A_{n}[u]\right\|_{\rho} \leq \frac{\Delta t}{\rho^{\prime}-\rho}\left[c R \varepsilon+\rho_{0} K\right]
$$

This shows that (v) is satisfied with $C_{2}=c R \varepsilon+K$, which $\rightarrow 0$ as the numerical parameters vanish, provided that $K \rightarrow 0$. Requiring that $K \rightarrow 0$ places obvious constraints on how the numerical parameters may be taken to 0 .

We are now almost ready to apply Theorem 4.1. It remains to determine $R, R_{0}$ and $\gamma$. It is natural to take

$$
\gamma=\omega
$$

As in the filtering case, the choices of $R$ and $R_{0}$ are a little delicate, since we want to ensure both that the method converges (i.e., $R \rightarrow 0$ ) and that the resulting $\lambda$ is a small perturbation of $\lambda_{0}$. One can check that if we take

$$
R_{0}=K \rho_{0}^{\omega} / \sqrt{\varepsilon} \text { and } R=K / \varepsilon \omega
$$

and require $K /(\varepsilon \omega)<1 / 2$ together with the appropriate smallness conditions on $\Delta t, h, \tau, \varepsilon_{r}$, then Theorem 4.1 yields

$$
\begin{aligned}
& \left\|D\left(s^{n+1}-\tilde{s}^{n+1}\right)\right\|_{\rho_{n+1}} \leq \frac{c_{\omega}}{\omega}\left(\Delta t+h+\frac{\tau^{1-\omega}}{\Delta t}+\frac{\varepsilon_{r}}{\tau}\right) \quad(\mathrm{PVM}) \\
& \left\|D\left(s^{n+1}-\tilde{s}^{n+1}\right)\right\|_{\rho_{n+1}} \leq \frac{c_{\omega}}{\omega}\left(\Delta t+e^{(1-\omega) \rho_{0} / h}+\frac{\tau^{1-\omega}}{\Delta t}+\frac{\varepsilon_{r}}{\tau}\right) \quad(\mathrm{MPVM})
\end{aligned}
$$

where

$$
\rho_{n+1}=\omega \rho_{0}-\lambda(n+1) \Delta t \quad \text { and } \quad \lambda=1 / 2+c_{\omega} \sqrt{\varepsilon}
$$

since $\lambda_{0}=\max \left(\lambda_{f}, \kappa / 2\right)=1 / 2+c \epsilon$ for some $c$. Finally, since $\left\|s^{n+1}-\tilde{s}^{n+1}\right\|_{\rho_{n+1}} \leq$ $\left\|D\left(s^{n+1}-\tilde{s}^{n+1}\right)\right\|_{\rho_{n+1}}$, this completes the proof of Theorem 3.2, which is the main result of this work. 


\section{Proof of the discrete Cauchy-Kowalewski theorem WITH ROUNDOFF ERROR AND FILTERING}

In this section, the proof of Theorem 4.2 is presented. This theorem provides uniform bounds for the numerical solution taking into account roundoff error and filtering. The proof of Theorem 4.2 is performed by carefully estimating each wavenumber separately and using induction.

Since the projection is applied to the right hand side of (4.9), the $n$th iterate $v_{n}$ satisfies

$$
\left|\hat{v}_{n}(k)\right|>\tau \quad \text { or } \quad \hat{v}_{n}(k)=0 .
$$

This implies that there are three cases for the next iterate:

(a) $\hat{v}_{n+1}(k)=0$,

(b) $\left|\hat{v}_{n+1}(k)\right|>\tau$ and $\hat{v}_{n}(k)=0$,

(c) $\left|\hat{v}_{n+1}(k)\right|>\tau$ and $\left|\hat{v}_{n}(k)\right|>\tau$.

Cases (b) and (c) can be used to estimate the size of the Fourier coefficients of the roundoff error in terms of the nonlinear term $A_{n}$ and the previous iterate $v_{n}$, respectively. Consider (b) first. This implies that

$$
\left|\widehat{A_{n}}\left[v_{n}\right](k)+\hat{e}_{r}(k)\right|>\tau / \Delta t
$$

Further, assumption (vi) implies that

$$
\left|\widehat{A_{n}}\left[v_{n}\right](k)\right|>\frac{\tau}{2 \Delta t} .
$$

Again using (vi), this implies that

$$
\left|\widehat{e}_{r}(k)\right|<\frac{2 \varepsilon_{r} \Delta t}{\tau}\left|\widehat{A_{n}}\left[v_{n}\right](k)\right| .
$$

For case (c), it is straightforward to see that

$$
\left|\hat{e}_{r}(k)\right|<\frac{\varepsilon_{r}}{\tau}\left|\hat{v}_{n}(k)\right|
$$

These bounds will be important in the proof.

Now, let $0<\gamma<1$, let $\Pi$ be a linear Fourier projection operator as described above, let $m \leq n$, let $\lambda$ be as in (4.13), and let $R_{2}, R_{1}$ be as in (4.10) and (4.11). Further, let $\rho$ be such that

$$
0<\rho<\rho_{0}-\lambda m \Delta t .
$$

Suppose, by way of induction, that

$$
\begin{aligned}
\left\|v_{m}\right\|_{\rho} & \leq R, \\
\left(\rho_{0}-\rho-\lambda m \Delta t\right)^{\gamma}\left\|A_{m}\left[\Pi v_{m}\right]\right\|_{\rho} & \leq R_{2}, \\
\left(\rho_{0}-\rho-\lambda m \Delta t\right)^{\gamma}\left\|(L-I) v_{m}\right\|_{\rho} & \leq \Delta t \cdot R_{1}
\end{aligned}
$$

for any Fourier filter $\Pi$. It is straightforward to see that (6.7)-(6.9) hold at $m=0$ by assumption (viii) on the initial data and the definitions of $R_{2}$ and $R_{1}$. The proof will be complete when it is shown that (6.7)-(6.9) hold for $m=n+1$.

The bound (6.7) will be established first. For each wavenumber $k$, define $m_{k}$ to be the largest integer such that

$$
0 \leq m_{k} \leq n+1 \quad \text { and } \quad \hat{v}_{m_{k}}(k)=0 .
$$


On the other hand, if

$$
\hat{v}_{m}(k) \neq 0 \quad \text { for all } 0 \leq m \leq n+1,
$$

then set $m_{k}=0$. Given $m_{k}$, the solution $v_{n+1}$ can be written as follows:

$$
\hat{v}_{n+1}(k)=\sum_{m=m_{k}}^{n} l^{n-m}\left(\hat{v}_{m+1}-l \hat{v}_{m}\right)+l^{n+1-m_{k}} \hat{v}_{m_{k}},
$$

where $\widehat{L}=l$. Roughly speaking, $m_{k}$ measures the amount of time a mode is inactive. Note that if $m_{k} \neq 0$, then the last term in (6.12) vanishes. Each Fourier mode of $v_{n+1}$ will be estimated separately to establish (6.7). In this analysis, the effect of the filter $P$ will be written out explicitly, so that the nonlinearity of $P$ can be handled. There are now two cases to consider.

Case 1. $\hat{v}_{n+1}(k)=0$. In this case, the bound is trivial.

Case 2. $\left|\hat{v}_{n+1}(k)\right|>\tau$. Estimate each term in (6.12). Since $\left|\hat{v}_{m+1}(k)\right|>\tau$, then the filter has no effect and the equation for $\hat{v}_{m+1}$ is

$$
\hat{v}_{m+1}=l \hat{v}_{m}+\Delta t\left(\widehat{A}_{m}\left[v_{m}\right]+\hat{e}_{r}\right) .
$$

Consider first the subcase $n \geq m>m_{k}$ or $n \geq m \geq m_{k}$ if $m_{k}=0$. This implies that

$$
\left|\hat{v}_{m}(k)\right|>\tau,
$$

since otherwise the solutions would be set equal to zero by the filter. Therefore, case (c) applies, to give

$$
\begin{aligned}
\left|\hat{v}_{m+1}-l \hat{v}_{m}\right| & \leq \Delta t\left(\left|\widehat{A_{m}}\left[v_{m}\right]\right|+\left|\hat{e}_{r}\right|\right) \\
& \leq \Delta t\left(\left|\widehat{A_{m}}\left[v_{m}\right]\right|+\frac{\varepsilon_{r}}{\tau}\left|\hat{v}_{m}\right|\right) .
\end{aligned}
$$

Now, consider the subcase $m=m_{k}$ with $m_{k} \neq 0$. This implies that

$$
\left|\hat{v}_{m_{k+1}}\right|>\tau \text { and } \hat{v}_{m}=0,
$$

which is exactly case (b). This implies that

$$
\begin{aligned}
\left|\hat{v}_{m_{k+1}}-l \hat{v}_{m_{k}}\right| & \leq \Delta t\left(\left|\widehat{A_{m_{k}}}\left[v_{m_{k}}\right]\right|+\left|\hat{e}_{r}\right|\right) \\
& \leq \Delta t\left(1+\frac{2 \varepsilon_{r} \Delta t}{\tau}\right)\left|\widehat{A_{m_{k}}}\left[v_{m_{k}}\right]\right| .
\end{aligned}
$$

Finally, it remains to consider the term $\hat{v}_{m_{k}}$. If $m_{k}=0$, then this term is equal to 0 . Otherwise, it is $\hat{v}_{0}$. This completes the analysis of Case 2 .

Now, combining the two cases and (6.12), (6.15) and (6.17), an overestimate for (6.12) is obtained:

$$
\left|\hat{v}_{n+1}(k)\right| \leq \Delta t \sum_{m=0}^{n}|l|^{n-m}\left[\left(1+\frac{2 \varepsilon_{r} \Delta t}{\tau}\right)\left|\widehat{A_{m}}\left[v_{m}\right](k)\right|+\frac{\varepsilon_{r}}{\tau}\left|\hat{v}_{m}(k)\right|\right]+|l|^{n+1}\left|\hat{v}_{0}\right| .
$$

If we recall that the $\rho$-norm is given by

$$
\|v\|_{\rho}=\sum_{k} e^{\rho|k|}|\hat{v}(k)|,
$$


then (6.18) implies that

$$
\begin{aligned}
\left\|v_{n+1}\right\|_{\rho} \leq & \Delta t \sum_{m=0}^{n}\left[\left(1+\frac{2 \varepsilon_{r} \Delta t}{\tau}\right)\left\|L^{n-m} A_{m}\left[v_{m}\right]\right\|_{\rho}+\frac{\varepsilon_{r}}{\tau}\left\|L^{n-m} v_{m}\right\|_{\rho}\right]+\left\|L^{n+1} v_{0}\right\|_{\rho} \\
\leq & +\Delta t \sum_{m=0}^{n}\left[\left(1+\frac{2 \varepsilon_{r} \Delta t}{\tau}\right)\left\|A_{m}\left[v_{m}\right]\right\|_{\rho+\lambda_{0}(n-m) \Delta t}+\frac{\varepsilon_{r}}{\tau}\left\|v_{m}\right\|_{\rho+\lambda_{0}(n-m) \Delta t}\right] \\
& +\left\|v_{0}\right\|_{\rho+\lambda_{0}(n+1) \Delta t},
\end{aligned}
$$

using assumption (i). Let $\rho \leq \rho_{n}=\rho_{0}-\lambda n \Delta t$. Since $\lambda>\lambda_{0}$, then $\rho+\lambda_{0}(n-m) \Delta t<$ $\rho_{0}-\lambda m \Delta t$ for $m \leq n$. So, the induction hypothesis (6.8) implies that

$$
\left\|A_{m}\left[v_{m}\right]\right\|_{\rho+\lambda_{0}(n-m) \Delta t} \leq \frac{R_{2}}{\left[\rho_{0}-\left(\rho+\lambda_{0}(n-m) \Delta t\right)-\lambda m \Delta t\right]^{\gamma}} .
$$

Writing $\lambda=\lambda_{0}+\lambda^{\prime}$ and using (6.21) and the induction hypothesis (6.7) in (6.20) gives

$$
\left\|v_{n+1}\right\|_{\rho} \leq\left(1+\frac{2 \varepsilon_{r} \Delta t}{\tau}\right) R_{2} \Delta t \sum_{m=0}^{n} \frac{1}{\left(\rho_{0}-\rho-\lambda_{0} n \Delta t-\lambda^{\prime} m \Delta t\right)^{\gamma}}+\left(\delta+\frac{\varepsilon_{r}}{\tau} t\right) R
$$

in which $t=n \Delta t$, where we have also used assumption (viii). Estimate the sum in (6.22) by the integral inequality

$$
\begin{aligned}
\Delta t \sum_{m=0}^{n} \frac{1}{\left(\rho_{0}-\rho-\lambda_{0} n \Delta t-\lambda^{\prime} m \Delta t\right)^{\gamma}} & \leq \int_{0}^{n \Delta t}\left(\rho_{0}-\rho-\lambda_{0} n \Delta t-\lambda^{\prime} t\right)^{-\gamma} d t \\
& \leq \frac{\rho_{0}^{1-\gamma}}{\lambda^{\prime}(1-\gamma)}
\end{aligned}
$$

Now, using (6.23) in (6.22) gives

$$
\left\|v_{n+1}\right\|_{\rho} \leq\left(1+\frac{2 \varepsilon_{r} \Delta t}{\tau}\right) \frac{R_{2} \rho_{0}^{1-\gamma}}{\lambda^{\prime}(1-\gamma)}+\left(\delta+\frac{\varepsilon_{r}}{\tau} t\right) R
$$

Therefore, since

$$
\lambda^{\prime}=\lambda-\lambda_{0} \geq\left(1+\frac{2 \varepsilon_{r} \Delta t}{\tau}\right)\left(1-\delta-\frac{\varepsilon_{r}}{\tau} t\right)^{-1} \frac{R_{2} \rho^{1-\gamma}}{R(1-\gamma)}
$$

by (4.13), the estimate

$$
\left\|v_{n+1}\right\|_{\rho_{n}} \leq R
$$

is proved. Since $\rho_{n+1}<\rho_{n}$, this proves the induction hypothesis $(6.7)$ for $m=n+1$, and in fact proves the theorem once the $n+1$ induction step is proved for $(6.8)$ and (6.9).

So we turn to the proof of (6.8) for $m=n+1$. Begin by defining $\rho^{\prime}$ to be

$$
\rho^{\prime}=\frac{1}{2}\left(\rho_{0}-\lambda n \Delta t+\rho\right)
$$

for any $\rho$. Thus,

$$
0<\rho<\rho_{0}-\lambda(n+1) \Delta t \Rightarrow \rho<\rho^{\prime}<\rho_{0}-\lambda n \Delta t .
$$


Further, for any Fourier projection $\Pi$ define the Fourier projections $\Pi_{n+1}$ and $\Pi^{\prime}$ by

$$
\begin{gathered}
\widehat{\Pi}_{n+1}= \begin{cases}0 & \text { if } \hat{v}_{n+1}=0, \\
1 & \text { otherwise },\end{cases} \\
\Pi^{\prime}=\Pi_{n+1} \Pi .
\end{gathered}
$$

Since $\rho^{\prime}<\rho_{n},(6.26)$ implies that $\left\|v_{n+1}\right\|_{\rho^{\prime}}<R$. Thus we may apply (v), as well as (iii) and the induction hypothesis (6.8) for $\Pi^{\prime}$ on $v_{n}$, to obtain

$$
\begin{aligned}
\| A_{n+1} & {\left[\Pi v_{n+1}\right]\left\|_{\rho}=\right\| A_{n+1}\left[\Pi^{\prime} v_{n+1}\right] \|_{\rho} } \\
\leq & \left\|A_{n}\left[\Pi^{\prime} v_{n}\right]\right\|_{\rho}+\left\|A_{n+1}\left[\Pi^{\prime} v_{n+1}\right]-A_{n}\left[\Pi^{\prime} v_{n+1}\right]\right\|_{\rho} \\
& +\left\|A_{n}\left[\Pi^{\prime} v_{n+1}\right]-A_{n}\left[\Pi^{\prime} v_{n}\right]\right\|_{\rho} \\
\leq & \left\|A_{n}\left[\Pi^{\prime} v_{n}\right]\right\|_{\rho}+\frac{C_{1}}{\rho^{\prime}-\rho}\left\|\Pi^{\prime} v_{n+1}-\Pi^{\prime} v_{n}\right\|_{\rho^{\prime}}+\frac{C_{2} \Delta t}{\rho^{\prime}-\rho} .
\end{aligned}
$$

The middle term is handled by estimating each Fourier mode separately as follows. There are two cases.

Case 1. $\hat{v}_{n+1}(k)=0$. By the definition of $\Pi^{\prime}$, this implies that

$$
\widehat{\Pi}^{\prime} v_{n+1}(k)-\widehat{\Pi}^{\prime} v_{n}(k)=0,
$$

so the bound is trivial.

Case 2. $\hat{v}_{n+1}(k) \neq 0$. This implies that $\left|\hat{v}_{n+1}(k)\right|>\tau$, so that

$$
\hat{v}_{n+1}(k)=l \hat{v}_{n}(k)+\Delta t \widehat{A_{n}}\left[v_{n}\right](k)+\Delta t \hat{e}_{r}(k) .
$$

For such $k$,

$$
\hat{v}_{n+1}(k)-\hat{v}_{n}(k)=(l-1) \hat{v}_{n}(k)+\Delta t \widehat{A_{n}}\left[v_{n}\right](k)+\Delta t \hat{e}_{r}(k) .
$$

The roundoff error $\hat{e}_{r}(k)$ is estimated by combining cases (b) and (c) to give

$$
\left|\hat{e}_{r}(k)\right| \leq \frac{\varepsilon_{r}}{\tau}\left(\left|\hat{v}_{n}(k)\right|+2 \Delta t\left|\widehat{A_{n}}\left[v_{n}\right](k)\right|\right) .
$$

Now, combine (6.34) and (6.35) and use the linearity of $\Pi^{\prime}$ to estimate the middle term in (6.31) by

$$
\begin{aligned}
& \left\|\Pi^{\prime} v_{n+1}-\Pi^{\prime} v_{n}\right\|_{\rho^{\prime}} \leq\left\|v_{n+1}-v_{n}\right\| \rho^{\prime} \\
& \quad \leq\left\|(L-I) v_{n}\right\|_{\rho^{\prime}}+\Delta t\left(1+\frac{2 \varepsilon_{r} \Delta t}{\tau}\right)\left\|A_{n}\left[v_{n}\right]\right\|_{\rho^{\prime}}+\frac{\varepsilon_{r} \Delta t}{\tau}\left\|v_{n}\right\|_{\rho^{\prime}} .
\end{aligned}
$$

This completes the analysis of Case 2 .

Applying the induction hypotheses (6.7)-(6.9) and the two cases with the definition of $\rho^{\prime}$ from (6.27) to (6.31) we get

$$
\begin{aligned}
\left\|A_{n+1}\left[\Pi v_{n+1}\right]\right\|_{\rho} \leq & R_{2}\left(\rho_{0}-\rho-\lambda n \Delta t\right)^{-\gamma} \\
& +\left[C_{1} \frac{2 \varepsilon_{r} \Delta t}{\tau} R+2 C_{2} \Delta t\right]\left(\rho_{0}-\rho-\lambda n \Delta t\right)^{-1} \\
& +\Delta t C_{1} 2^{1+\gamma}\left[R_{1}+\left(1+\frac{2 \varepsilon_{r} \Delta t}{\tau}\right) R_{2}\right]\left(\rho_{0}-\rho-\lambda n \Delta t\right)^{-1-\gamma}
\end{aligned}
$$


Now, another integral inequality implies that

$$
\left(\rho_{0}-\rho-\lambda(n+1) \Delta t\right)^{-\gamma} \geq\left(\rho_{0}-\rho-\lambda n \Delta t\right)^{-\gamma}+\gamma \lambda \Delta t\left(\rho_{0}-\rho-\lambda n \Delta t\right)^{-1-\gamma} .
$$

Using (6.38) in (6.37) and the inequality

$$
\gamma \lambda \geq C_{1} 2^{1+\gamma}\left[\frac{R_{1}}{R_{2}}+\left(1+\frac{2 \varepsilon_{r} \Delta t}{\tau}\right)\right]+2 C_{2} \frac{\rho_{0}^{\gamma}}{R_{2}}+C_{1} \frac{2 \varepsilon_{r}}{\tau} \frac{R \rho_{0}^{\gamma}}{R_{2}},
$$

as guaranteed by (4.13), yields the bound

$$
\left(\rho_{0}-\rho-\lambda(n+1) \Delta t\right)^{\gamma}\left\|A_{n+1}\left[\Pi v_{n+1}\right]\right\|_{\rho} \leq R_{2}
$$

with $0<\rho<\rho_{0}-\lambda(n+1) \Delta t$. This proves the $n+1$ induction step for (6.8).

It now remains to prove (6.9) for $m=n+1$. Again, we estimate each wavenumber individually. As before, there are two cases.

Case 1. $\hat{v}_{n+1}(k)=0$. This implies that $(l-1) \hat{v}_{n+1}(k)=0$, and the bound is trivial.

Case 2. $\hat{v}_{n+1}(k) \neq 0$. As before, this implies that

$$
\hat{v}_{n+1}(k)=l \hat{v}_{n}(k)+\Delta t \widehat{A_{n}}\left[v_{n}\right](k)+\Delta t \hat{e}_{r}(k) .
$$

This implies that

$$
(l-1) \hat{v}_{n+1}(k)=(l-1) l \hat{v}_{n}(k)+\Delta t(l-1) \widehat{A}_{n}\left[v_{n}\right](k)+\Delta t(l-1) \hat{e}_{r}(k) .
$$

Now, using (b) and (c) gives the bound

$$
\begin{aligned}
\left|(l-1) \hat{v}_{n+1}(k)\right| \leq & \left|(l-1) l \hat{v}_{n}(k)\right| \\
& +\Delta t\left(1+\frac{2 \varepsilon_{r} \Delta t}{\tau}\right)\left|(l-1) \widehat{A}_{n}\left[v_{n}\right](k)\right|+\frac{\varepsilon_{r} \Delta t}{\tau}\left|(l-1) \hat{v}_{n}(k)\right| .
\end{aligned}
$$

This completes the analysis of Case 2 .

Combining Cases 1 and 2, the induction hypotheses, assumption (i) and the definition of $\rho^{\prime}$ gives us the bound

$$
\begin{aligned}
\left\|(L-I) v_{n+1}\right\|_{\rho} \leq & \Delta t R_{1}\left(\rho_{0}-\rho-\left(\lambda n+\lambda_{0}\right) \Delta t\right)^{-\gamma}+\frac{\varepsilon_{r} \Delta t^{2}}{\tau} R_{1}\left(\rho_{0}-\rho-\lambda n \Delta t\right)^{-\gamma} \\
& +\Delta t^{2} \lambda_{0} R_{2} 2^{1+\gamma}\left(1+\frac{2 \varepsilon_{r} \Delta t}{\tau}\right)\left(\rho_{0}-\rho-\lambda n \Delta t\right)^{-1-\gamma} .
\end{aligned}
$$

Another integral inequality gives

$$
\begin{aligned}
\left(\rho_{0}-\rho-\lambda(n+1) \Delta t\right)^{-\gamma} \geq & \left(\rho_{0}-\rho-\left(\lambda n+\lambda_{0}\right) \Delta t\right)^{-\gamma} \\
& +\Delta t \gamma\left(\lambda-\lambda_{0}\right)\left(\rho_{0}-\rho-\left(\lambda n+\lambda_{0}\right) \Delta t\right)^{-1-\gamma} .
\end{aligned}
$$

Thus, using (6.45) in (6.44) as well as the fact that

$$
\lambda \geq \lambda_{0}+\frac{\lambda_{0} R_{2}}{\gamma R_{1}}\left(1+\frac{2 \varepsilon_{r} \Delta t}{\tau}\right) 2^{\gamma+1}+\frac{\varepsilon_{r}}{\gamma \tau} \rho_{0},
$$

as guaranteed by (4.13), we get the bound

$$
\left(\rho_{0}-\rho-\lambda(n+1) \Delta t\right)^{\gamma}\left\|(L-I) v_{n+1}\right\|_{\rho} \leq \Delta t \cdot R_{1}
$$

with $0<\rho<\rho_{0}-\lambda(n+1) \Delta t$. This proves the $n+1$ induction step for (6.9) and completes the proof of Theorem 4.2. 


\section{Appendix 1: Abstract Cauchy-Kowalewski theorem, CONTINUOUS VERSION}

In this appendix, a time continuous version of the abstract Cauchy-Kowalewski theorem is presented. This version is a modification of the strengthened formulation of Safonov [21] and is specialized for perturbative problems. See also [18, 19, 7]. Consider the equation

$$
u(\alpha, t)=\varepsilon \int_{0}^{t} \tilde{N}[u]\left(\alpha, t, t^{\prime}\right) d t^{\prime} .
$$

Let $B_{\rho}$ be a family of Banach spaces for $0<\rho<\rho_{0}$ with norm $\|\cdot\|_{\rho}$ such that $B_{\rho^{\prime}} \subset B_{\rho}$ and $\|u\|_{\rho} \leq\|u\|_{\rho^{\prime}}$ for $0<\rho<\rho^{\prime}$. Suppose that $\widetilde{N}$ satisfies the following assumptions:

(A) For any $0<\rho<\rho^{\prime}<\rho_{0}-\lambda_{0} t$ and $t \geq t^{\prime}$,

$$
\left\|\tilde{N}[u]\left(\cdot, t, t^{\prime}\right)\right\|_{\rho} \leq\|N[u](\cdot, t)\|_{\rho+\lambda_{0}\left(t-t^{\prime}\right)},
$$

where $N$ is a continuous mapping from $\left\{u \in B_{\rho^{\prime}},\|u\|_{\rho^{\prime}} \leq R\right\}$ into $B_{\rho}$.

Moreover, $N$ satisfies

(B) For any $0<\rho<\rho^{\prime}<\rho_{0}-\lambda_{0} t$ and for any $u, v$ with $u, v \in B_{\rho^{\prime}}$ and $\|u\|_{\rho^{\prime}},\|v\|_{\rho^{\prime}} \leq R$, there exists a constant $C$ such that

$$
\|N[u](\cdot, t)-N[v](\cdot, t)\|_{\rho} \leq \frac{C}{\rho^{\prime}-\rho}\|u-v\|_{\rho^{\prime}},
$$

where $C$ is independent of $u, v, \rho, \rho^{\prime}, t$.

(C) Finally, suppose that $N[0](\alpha, t)$ is a continuous function of $t$ for $0 \leq t<\rho_{0} / \lambda_{0}$ with values in $B_{\rho}$ for $\rho<\rho_{0}$ such that

$$
\|N[0](\cdot, t)\|_{\rho} \leq K
$$

for $\rho<\rho_{0}-\lambda_{0} t$ and some $K$ independent of $t$ and $\rho$.

Theorem 7.1 (Abstract Cauchy-Kowalewski Theorem). For any $R, K, C, \rho_{0}, \lambda_{0}$ and $0<\beta<1$ such that $(\mathrm{A})-(\mathrm{C})$ are satisfied, there exist $\lambda$ and a unique solution $u$ to (7.1) such that

$$
\|u\|_{\rho} \leq R
$$

for $0<\rho<\rho_{0}-\lambda t$ and

$$
\lambda=\max \left\{\lambda_{0}+\frac{\varepsilon R_{0} \rho_{0}^{1-\beta}}{R(1-\beta)}, \lambda_{0}+2^{2+\beta} \frac{C \varepsilon}{\beta^{2}}\right\},
$$

where $R_{0} \geq K \rho_{0}^{\gamma}$ and $C$ is the constant in assumption (B).

The proof of Theorem 7.1 closely follows that presented in [21], and we do not give it here.

Finally, this theorem can be applied to the nonlinear model problems (2.13) and (2.16) to show existence of solutions as follows. Take the analytic norm

$$
\|f\|_{\rho}=\sum_{k} e^{\rho|k|}\left|\hat{f}_{k}\right|
$$

and differentiate (2.13) and (2.16) to obtain the equations in quasi-linear form. Further, shift the solutions by $u$ and $u-w+F$, respectively. That is, take the new 
variable $\chi=\eta_{\alpha}-u_{\alpha}$ for (2.13) and $\chi=\nu_{\alpha}-u_{\alpha}+w_{\alpha}-F_{\alpha}$ for (2.16). In addition, suppose that the mean of the initial data $\eta(0, t)$ and $\nu(0, t)$ is exactly 0 . Then,

$$
\widetilde{N}[\chi]=\partial_{\alpha} \widetilde{A}\left[\partial_{\alpha}^{-1} \chi+u\right], \quad \text { where } \partial_{\alpha}^{-1} \chi=\eta-u,
$$

for $(2.13)$ and

$$
\widetilde{N}[\chi]=\partial_{\alpha} \widetilde{A}\left[\partial_{\alpha}^{-1} \chi+u-w+F\right], \quad \text { where } \partial_{\alpha}^{-1} \chi=\nu-u+w-F,
$$

for (2.16). Then, it can be shown that assumptions (A)-(C) are satisfied for some $R, C, K$ and with $\rho_{0}$ replaced by $\gamma_{1} \rho_{0}$ for $(2.13)$ and $\gamma_{2} \rho_{0}$ for $(2.16)$, where $0<\gamma_{1}<$ $\gamma_{2}<1$. The reason for this is that the initial conditions given for these equations in Section 2 are in $B_{\rho}$ for $\rho<\rho_{0}$, i.e., $\eta_{k}(0)=e^{-\rho_{0}|k|}$ is not in $B_{\rho_{0}}$.

\section{Appendix 2: Proof of filtering lemmas}

In this section, the proofs of Lemmas 5.1-5.3 are given.

Proof of Lemma 5.1. By the definition of $P$, we have

$$
\widehat{(I-P) f_{k}}= \begin{cases}\hat{f}_{k}, & \text { if }\left|\hat{f}_{k}\right|<\tau \\ 0, & \text { if }\left|\hat{f}_{k}\right| \geq \tau .\end{cases}
$$

To bound $\left|\hat{f}_{k}\right|$ when $\left|\hat{f}_{k}\right|<\tau$, use $\left|\hat{f}_{k}\right|<\|f\|_{\rho^{\prime}} e^{-\rho^{\prime}|k|}$ if $e^{-\rho^{\prime}|k|}<\sigma$ (i.e., if $|\log \sigma| / \rho^{\prime}<|k|$ ), and use $\left|\hat{f}_{k}\right|<\tau$ if $\sigma<e^{-\rho^{\prime}|k|}$ (i.e., if $|\log \sigma| / \rho^{\prime}>|k|$ ). It follows that

$$
\begin{aligned}
\|(I-P) f\|_{\rho} & \leq \sum_{\left|\hat{f}_{k}\right|<\tau}\left|\hat{f}_{k}\right| e^{\rho|k|} \\
& \leq \sum_{|\log \sigma| / \rho^{\prime}<|k|}\|f\|_{\rho^{\prime}} e^{\left(\rho-\rho^{\prime}\right) / k}+\sum_{|k|<|\log \sigma| / \rho^{\prime}} \tau e^{\rho|k|} \\
& \leq\|f\|_{\rho^{\prime}} \frac{a^{n}}{1-a}+\tau \frac{b^{n+1}-1}{b-1} \\
& \leq\|f\|_{\rho^{\prime}} \sigma^{1-\rho / \rho^{\prime}}\left(1+\left(\rho^{\prime}-\rho\right)^{-1}\right)+\tau \sigma^{-\rho / \rho^{\prime}}\left(1+\rho^{-1}\right),
\end{aligned}
$$

in which

$$
n=|\log \sigma| / \rho^{\prime}, \quad a=e^{\left(\rho-\rho^{\prime}\right)}, \quad b=e^{\rho} .
$$

Choose $\sigma=\tau /\|f\|_{\rho^{\prime}}$, to obtain

$$
\|(I-P) f\|_{\rho} \leq\left(2+\rho^{-1}+\left(\rho^{\prime}-\rho\right)^{-1}\right)\|f\|_{\rho^{\prime}}^{\rho / \rho^{\prime}} \tau^{1-\rho / \rho^{\prime}} .
$$

This proves Lemma 5.1. If $\tau=\tau(k)$, then in the above $\tau=\max _{k} \tau(k)$.

Proof of Lemma 5.2. Decompose the sum $\left\|P\left(f+e_{r}\right)-P f\right\|_{\rho}$ into three parts, as

$$
\begin{aligned}
\left\|P\left(f+e_{r}\right)-P f\right\|_{\rho} & \\
& =\left(\sum_{\left|\hat{f}_{k}\right|>2 \tau}+\sum_{\left|\hat{f}_{k}\right|<\tau / 2}+\sum_{\tau / 2<\left|\hat{f}_{k}\right|<2 \tau}\right) e^{\rho|k|} \widehat{\mid P\left(f+e_{r}\right)}(k)-\widehat{P f}(k) \mid .
\end{aligned}
$$


In the first sum, $\widehat{P\left(f+e_{r}\right)}(k)-\widehat{P f}(k)=\hat{e}_{r}(k)$, so that $\left|\widehat{P\left(f+e_{r}\right)}(k)-\widehat{P f}(k)\right| \leq$ $\varepsilon_{r}$. Moreover, $2 \tau<\left|\hat{f}_{k}\right| \leq\|f\|_{\rho^{\prime}} e^{-\rho^{\prime}|k|}$, so that $|k| \leq k_{1}=\frac{1}{\rho^{\prime}} \log \left(\|f\|_{\rho^{\prime}} / 2 \tau\right)$. Thus

$$
\begin{aligned}
& \sum_{\left|\hat{f}_{k}\right|>2 \tau} e^{\rho|k|}\left|\widehat{P\left(f+e_{r}\right)}(k)-\widehat{P f}(k)\right| \leq \varepsilon_{r} \sum_{k \leq k_{1}}\left(\|f\|_{\rho^{\prime}} / 2 \tau\right)^{\rho / \rho^{\prime}} \\
& \quad \leq \frac{\varepsilon_{r}}{\rho^{\prime}}\left(\|f\|_{\rho^{\prime}} / 2 \tau\right)^{\rho / \rho^{\prime}} \log \left(\|f\|_{\rho^{\prime}} / 2 \tau\right) .
\end{aligned}
$$

A cruder bound on this sum is

$$
\begin{aligned}
\sum_{\left|\hat{f}_{k}\right|>2 \tau} e^{\rho|k|} \varepsilon_{r} & \leq \frac{\varepsilon_{r}}{2 \tau} \sum e^{\rho|k|}\left|\hat{f}_{k}\right| \leq \frac{\varepsilon_{r}}{2 \tau}\|f\|_{\rho^{\prime}} \sum e^{-\left(\rho^{\prime}-\rho\right)|k|} \\
& \leq \frac{\varepsilon_{r}}{2 \tau}\left(1+\left(\rho^{\prime}-\rho\right)^{-1}\right)\|f\|_{\rho^{\prime}} .
\end{aligned}
$$

In the second sum, $\left.\widehat{P\left(f+e_{r}\right.}\right)(k)=\widehat{P f}(k)=0$. Thus, it contributes nothing.

In the third sum, a straightforward argument shows that $\widehat{P\left(f+e_{r}\right)}(k)-\widehat{P f}(k) \mid \leq$ $2 \tau \leq 4\left|\hat{f}_{k}\right|=4\left|\left(\hat{f}-\widehat{P_{2 \tau} f}\right)(k)\right|$. Thus

$$
\sum_{\tau / 2<\left|\hat{f}_{k}\right|<2 \tau} \leq 4\left\|f-P_{2 \tau} f\right\|_{\rho}
$$

This proves Lemma 5.2. If $\tau=\tau(k)$, then in the above $\tau=\min _{k} \tau(k)$ except in the projection, where the dependence of $\tau$ upon $k$ is kept.

Proof of Lemma 5.3. Decompose the norm $\|P f-P g\|$ into four parts, as

$$
\begin{aligned}
\|P f-P g\|_{\rho}= & \|P f-P g\|_{\rho}^{(1)}+\|P f-P g\|_{\rho}^{(2)} \\
& +\|P f-P g\|_{\rho}^{(3)}+\|P f-P g\|_{\rho}^{(4)}
\end{aligned}
$$

in which

$$
\|P f-P g\|_{\rho}^{(i)}=\sum_{k \in K_{i}} \widehat{P^{f}}(k)-\widehat{P^{g}}(k) \mid e^{\rho|k|}
$$

with

$$
\begin{aligned}
& K_{1}=\{k:|\hat{f}(k)|>\tau,|\hat{g}(k)|>\tau \mid\}, \\
& K_{2}=\{k:|\hat{f}(k)|<\tau,|\hat{g}(k)|<\tau \mid\}, \\
& K_{3}=\{k:|\hat{f}(k)|<\tau,|\hat{g}(k)|>\tau \mid\}, \\
& K_{4}=\{k:|\hat{f}(k)|>\tau,|\hat{g}(k)|<\tau \mid\} .
\end{aligned}
$$

For $k \in K_{1}$, we have $P f=f$ and $P g=g$, so that

$$
\|P f-P g\|_{\rho}^{(1)}=\|f-g\|_{\rho}^{(1)} .
$$


For $k \in K_{2}$, we have $P f=0$ and $P g=0$, so that

$$
\|P f-P g\|_{\rho}^{(2)}=0 .
$$

For $k \in K_{3}$, we have $P f=0$ and $P g=g$, so that

$$
\begin{aligned}
\| P f- & P g\left\|_{\rho}^{(3)}=\right\| g\left\|_{\rho}^{(3)} \leq\right\| f-g\left\|_{\rho}^{(3)}+\right\| f-P f \|_{\rho}^{(3)} \\
& \leq\|f-g\|_{\rho}^{(3)}+\left(\|f\|_{\rho^{\prime}}^{(3)}\right)^{\rho / \rho^{\prime}}\left(2+\rho^{-1}+\left(\rho^{\prime}-\rho\right)^{-1}\right) \tau^{1-\rho / \rho^{\prime}} .
\end{aligned}
$$

For $k \in K_{4}$, we have

$$
\|P f-P g\|_{\rho}^{(4)}=\|f\|_{\rho}^{(4)}=S_{1}+S_{2},
$$

in which $S_{1}$ is the sum over $k \in K_{4}$ satisfying $e^{-\rho^{\prime}|k|}<\sigma$ and $S_{2}$ is the sum over $k \in$ $K_{4}$ satisfying $e^{-\rho^{\prime}|k|}>\sigma$. If $k \in K_{4}$ and $|k|>|\log \sigma| / \rho^{\prime}$, then $|\hat{f}(k)|<\|f\|_{\rho^{\prime}}^{(4)} e^{-\rho^{\prime}|k|}$ and

$$
\begin{aligned}
S_{1} & \leq\|f\|_{\rho^{\prime}}^{(4)} \sum_{|k|>|\log \sigma| / \rho^{\prime}} e^{-(\rho-\rho)|k|} \\
& \leq\|f\|_{\rho^{\prime}}^{(4)}\left(1+\left(\rho^{\prime}-\rho\right)^{-1}\right) \sigma^{1-\rho / \rho^{\prime}} .
\end{aligned}
$$

Similarly, for $k \in K_{4}$ we have $e^{-\rho^{\prime}|k|}>\sigma\left(\right.$ and $\left.\tau>\left|\hat{g}_{k}\right|\right)$, so that

$$
\begin{aligned}
S_{2} & \leq \sum_{e^{-\rho^{\prime}|k|}>\sigma, \tau>\left|\hat{g}_{k}\right|}\left(\left|\hat{f}_{k}-\hat{g}_{k}\right|+\tau\right) e^{\rho|k|} \\
& \leq\|f-g\|_{\rho}^{(4)}+\tau \sum_{|k|<|\log \sigma| / \rho^{\prime}} e^{\rho|k|} \\
& \leq\|f-g\|_{\rho}^{(4)}+\tau \sigma^{-\rho / \rho^{\prime}}\left(1+\rho^{-1}\right) .
\end{aligned}
$$

Choose $\sigma=\tau /\|f\|_{\rho^{\prime}}^{(4)}$ as above, to obtain

$$
\|P f-P g\|_{\rho}^{(4)} \leq\|f-g\|_{\rho}^{(4)}+\left(2+\rho^{-1}+\left(\rho^{\prime}-\rho\right)^{-1}\right)\|f\|_{\rho^{\prime}}^{(4) \rho / \rho^{\prime}} \tau^{1-\rho / \rho^{\prime}} .
$$

Combine these four sums together to obtain Lemma 5.3. If $\tau=\tau(k)$, then in the above $\tau=\max _{k} \tau(k)$.

\section{Appendix 3: Proof of time difference OF A NONLINEAR OPERATOR}

In this section, Lemma 5.4 is proved by explicitly analyzing the discrete Fourier transform of the nonlinear operator. We begin by writing

$$
F_{\mathrm{NL}}[f]=\sum_{m=2}^{\infty}(-1)^{m} F_{\mathrm{NL}}^{m}[f]
$$


where

$$
F_{\mathrm{NL}}^{m}[f]_{j}=\frac{h}{2 \pi i} \sum_{l \neq j} \frac{\left(f_{j}-f_{l}\right)^{m}}{\left(\alpha_{j}-\alpha_{l}\right)^{m+1}},
$$

using the PVM. Since analogous expressions can obtained for the MPVM, we focus on the PVM here. A straightforward computation shows that

$$
\widehat{F}_{\mathrm{NL}}[f]_{k}=\sum_{k_{1}+\cdots+k_{m}=k}\left(\prod_{r=1}^{m} \hat{f}_{k_{r}}\right) \widetilde{I}\left(k_{1}, \ldots, k_{m}, k\right),
$$

where

$$
\widetilde{I}\left(k_{1}, \ldots, k_{m}, k\right)=\frac{h}{2 \pi i} \sum_{l \neq 0} \frac{1}{\alpha_{l}} \prod_{r=1}^{m}\left(\frac{1-e^{i k_{r} \alpha_{l}}}{\alpha_{l}}\right) .
$$

Using the analysis presented in [8], it is straightforward to show that

$$
\left|\widetilde{I}\left(k_{1}, \ldots, k_{m}, k\right)\right| \leq \pi \prod_{r=1}^{m}\left|k_{r}\right|,
$$

where $c$ is independent of all $k_{r}$ and $m$. Consequently, one gets

$$
\begin{aligned}
\widehat{F}_{\mathrm{NL}}[f]_{k}-\widehat{F}_{\mathrm{NL}}[f-v]_{k}-\widehat{F}_{\mathrm{NL}}[g]_{k}+\widehat{F}_{\mathrm{NL}}[g-v]_{k} \\
=\sum_{k_{1}+\cdots+k_{m}=k} \tilde{I}\left(k_{1}, \ldots, k_{m}, k\right) \\
\cdot\left[\prod_{r=1}^{m} \hat{f}_{k_{r}}-\prod_{r=1}^{m}\left(\hat{f}_{k_{r}}-\hat{v}_{k_{r}}\right)-\prod_{r=1}^{m} \hat{g}_{k_{r}}+\prod_{r=1}^{m}\left(\hat{g}_{k_{r}}-\hat{v}_{k_{r}}\right)\right] .
\end{aligned}
$$

It is clear now that the terms $\prod_{r=1}^{m} \hat{f}_{k_{r}}, \prod_{r=1}^{m} \hat{g}_{k_{r}}, \prod_{r=1}^{m} \hat{v}_{k_{r}}$ cancel in the bracketed term on the RHS of (9.6), leaving only the cross terms remaining. For example, if $m=2$, this yields

$$
\hat{v}_{k_{2}}\left(\hat{f}_{k_{1}}-\hat{g}_{k_{1}}\right)+\hat{v}_{k_{1}}\left(\hat{f}_{k_{2}}-\hat{g}_{k_{2}}\right)
$$

and for $m=3$ one gets

$$
\begin{aligned}
\hat{v}_{k_{3}} \hat{f}_{k_{2}}\left(\hat{f}_{k_{1}}-\hat{g}_{k_{1}}\right) & +\hat{v}_{k_{3}} \hat{g}_{k_{1}}\left(\hat{f}_{k_{2}}-\hat{g}_{k_{2}}\right)-\hat{v}_{k_{1}} \hat{v}_{k_{2}}\left(\hat{f}_{k_{3}}-\hat{g}_{k_{3}}\right) \\
& +\hat{v}_{k_{1}}\left(\hat{f}_{k_{2}}-\hat{g}_{k_{2}}\right)\left(\hat{f}_{k_{3}}-\hat{v}_{k_{3}}\right)+\hat{v}_{k_{1}} \hat{g}_{k_{2}}\left(\hat{f}_{k_{3}}-\hat{g}_{k_{3}}\right) \\
& +\hat{v}_{k_{2}}\left[\left(\hat{f}_{k_{1}}-\hat{g}_{k_{1}}\right)\left(\hat{f}_{k_{3}}-\hat{v}_{k_{3}}\right)+\hat{g}_{k_{1}}\left(\hat{f}_{k_{3}}-\hat{g}_{k_{3}}\right)\right] .
\end{aligned}
$$

The higher order terms are handled analogously, and, after tedious computation, exact formulae can be obtained. 
Now, using (9.5) and (9.7), one gets

$$
\begin{aligned}
\| F_{\mathrm{NL}}[f]- & F_{\mathrm{NL}}[f-v]-F_{\mathrm{NL}}[g]+F_{\mathrm{NL}}[g-v] \|_{\rho} \\
\leq & c \sum_{|k|} e^{\rho|k|} \sum_{k_{1}+k_{2}=k}\left[\left|k_{1} \hat{v}_{k_{1}}\right| k_{2}\left(\hat{f}_{k_{2}}-\hat{g}_{k_{2}}\right)|+| k_{2} \hat{v}_{k_{2}}\left|k_{1}\left(\hat{f}_{k_{1}}-\hat{g}_{k_{1}}\right)\right|\right] \\
& +\sum_{|k|} e^{\rho|k|} \mid \text { H. O. T. } \mid \\
\leq & 2 c\|D v\|_{\rho}\|D(f-g)\|_{\rho}+\sum_{|k|} e^{\rho|k|} \mid \text { H. O. T. |, }
\end{aligned}
$$

where H. O. T. stands for the higher order terms. A straightforward but tedious computation shows that if $\|D v\|_{\rho} \leq R$ and $\|D f\|_{\rho},\|D f\|_{\rho} \leq \widetilde{R}$ with $R \leq \widehat{R} \leq \frac{1}{3}$, then the H.O.T. are estimated by

$$
\begin{aligned}
\sum_{|k|} e^{\rho|k|} \mid \text { H. O. T. } \mid & \leq c\|D f\|_{\rho}\|D(f-g)\|_{\rho} \sum_{m=3}^{\infty} \widetilde{R}^{m-2}\left[2(m-1)+(m+3) 2^{m-2}\right] \\
& \leq c\|D v\|_{\rho}\|D(f-g)\|_{\rho},
\end{aligned}
$$

where $c$ is independent of $v, f, g$, since

$$
\sum_{m=3}^{\infty} \widetilde{R}^{m-2}\left[2(m-1)+(m+3) 2^{m-2}\right] \leq \widetilde{R}\left[\frac{\widetilde{R}+6}{(1-\widetilde{R})^{2}}+\frac{28}{(1-2 \widetilde{R})^{2}}\right] \leq c .
$$

Finally, putting (9.9) together with (9.10) and (9.11) proves Lemma 5.4.

\section{REFERENCES}

1. G. R. Baker, Russel E. Caflisch, and Michael Siegel, Singularity formation during RayleighTaylor instability, Journal of Fluid Mechanics 252 (1993), 51-78. MR 94e:76034

2. J. T. Beale, T. Y. Hou, and J. Lowengrub, Growth rates for the linearized motion of fluid interfaces away from equilibrium, Comm. Pure Appl. Math. 46 (1993), 1269-1301. MR 95c:76016

3. larities in Fluids, Plasmas and Optics (R. C. Caflisch and G. Papanicolau, editors), NATO ASI Series, Kluwer, 1993, pp. 11-38.

4. $\_$Convergence of boundary integral methods for water waves, SIAM J. Num. Anal. 33 (1996), 1797-1843. MR 89b:76009

5. R. Caflisch and O. Orellana, Long time existence for a slightly perturbed vortex sheet, Comm. Pure Appl. Math. 39 (1986), 807-838. MR 87m:76018

6. Singular solutions and ill-posedness for the evolution of vortex sheets, SIAM J. Math. Anal. 20 (1989), 293-307. MR 90d:76020

7. R. E. Caflisch, A simplified version of the abstract Cauchy-Kowalewski Theorem with weak singularities, Bull. Amer. Math. Soc. 23 (1990), 495-500. MR 91f:35008

8. Russel E. Caflisch and John S. Lowengrub, Convergence of the vortex method for vortex sheets, SIAM J. Numer. Anal. 26 (1989), 1060-1080. MR 91g:76073

9. S. J. Cowley, G. R. Baker, and S. Tanveer, On the formation of Moore curvature singularities in vortex sheets, J. Fluid Mech., submitted.

10. W. S. Dai and M. J. Shelley, A numerical study of the effect of surface tension and noise on an expanding Hele-Shaw bubble, Phys. Fluids A 5 (1993), pp. 2131-2146. 
11. A. J. DeGregoria and L. W. Schwartz, A boundary integral method for two phase displacement in Hele-Shaw cells, J. Fluid Mech. 164 (1986), 383-400. MR 87h:76073

12. J. Duchon and R. Robert, Global vortex sheet solutions of Euler equations in the plane, J. Diff. Eqn. 73 (1988), 215-224. MR 89h:35262

13. H. Dym and H. McKean, Fourier Series and Integrals, Academic Press, New York, 1972. MR $56: 945$

14. T. Y. Hou, J. Lowengrub, and R. Krasny, Convergence of a point vortex method for vortex sheets, SIAM J. Numer. Anal. 28 (1991), 308-320. MR 92d:65153

15. R. Krasny, A study of singularity formation in a vortex sheet by the point-vortex approximation, J. Fluid Mechanics 167 (1986), 65-93. MR 87g:76028

16. D. I. Meiron, G. R. Baker, and S. A. Orszag, Analytic structure of vortex sheet dynamics, part 1, Kelvin-Helmholtz Instability, J. Fluid Mech. 114 (1982), 283-298. MR 83c:76017

17. D. W. Moore, The spontaneous appearance of a singularity in the shape of an evolving vortex sheet, Proc. Roy. Soc. London A 365 (1979), 105-119. MR 80b:76006

18. L. Nirenberg, An abstract form of the nonlinear Cauchy-Kowalewski theorem, J. Diff. Geom. 6 (1972), 561-576. MR 48:683

19. T. Nishida, A note on a theorem of Nirenberg, J. Diff. Geom. 12 (1977), 629-633. MR 80a:58013

20. P. G. Saffman and G. I. Taylor, The penetration of a fluid into a porous medium of a HeleShaw cell containing a more viscous fluid, Proc. Royal Soc. London Ser. A 245 (1958), 312329. MR 20:3697

21. M. V. Safonov, The abstract Cauchy-Kovalevskaya theorem in a weighted Banach space, Comm. Pure Appl. Math. 48 (1995), 629-637. MR 96g:35002

22. M. J. Shelley, A study of singularity formation in vortex sheet motion by a spectrally accurate vortex method, J. Fluid Mech. 244 (1992), 493-526. MR 93g:73065

23. C. Sulem, P. L. Sulem, C. Bardos, and U. Frisch, Finite time analyticity for the two and three dimensional Kelvin-Helmholtz instability, Comm. Math. Phys. 80 (1981), 485-516. MR 83d:76012

24. G. Tryggvason, Numerical simulations of the Rayleigh-Taylor problem, J. Computational Phys. 75 (1988), 253.

25. G. Tryggvason and H. Aref, Numerical experiments on Hele-Shaw flows with a sharp interface, J. Fluid Mech. 136 (1983), 1-30.

Department of Mathematics, UCLA, Box 951555, Los Angeles, California 90095-1555

Department of Applied Mathematics, California Institute of Technology, Pasadena, CALIFORNIA 91125

School of Mathematics, University of Minnesota, Minneapolis, Minnesota 55455

Current address: Department of Mathematics, University of North Carolina, Phillips Hall, Chapel Hill, North Carolina 27599 